\title{
Blends of Polybutadiene with Different Vinyl Contents and Polystyrene Studied with Small-Angle Neutron Scattering in Varying Temperature and Pressure Fields
}

\author{
Henrich Frielinghaus, ${ }^{t, \ddagger}$ Dietmar Schwahn, ${ }^{*, t}$ and Lutz Willnert \\ Forschungszentrum J ülich GmbH, Institut für Festkörperforschung, D-52425 J ülich, Germany, and \\ Risø National Laboratory, DK-4000 Roskilde, Denmark
}

Received J une 12, 2000; Revised Manuscript Received December 6, 2000

\begin{abstract}
Blends of polybutadiene with three different vinyl contents and polystyrene were studied by small-angle neutron scattering as a function of temperature and pressure. The data were analyzed with a crossover function combining the universality classes of the 3D-I sing and the mean field behavior and thus yield the spinodal temperature $T_{S}$, the Ginzburg number $\mathrm{Gi}$, and the critical amplitude $\mathrm{C}_{\mathrm{MF}}$. From these parameters the mean field Flory-Huggins interaction parameter is calculated in terms of entropic and enthal pic contributions. The application of the theory of random copolymers delivers large enthal pic and entropic contributions of the intramolecular interaction between the 1,2- and 1,4-butadiene units. These numbers could not be confirmed from measurements of the corresponding intermolecular interaction contributions in an h-PB $(1,2) / d-P B(1,4)$ blend, which thus leads to speculations about the equivalence of inter- and intramolecular interactions in random copolymers. The Ginzburg number proved to be specific to the vinyl content and thus deviated from the universal behavior $\mathrm{Gi} \propto \mathrm{V}^{-1}$ predicted by deGennes. By our pressure experiments we find that the Clausius-Clapeyron equation suggests the phase boundary to be influenced by the Flory-Huggins parameter and the Ginzburg number.
\end{abstract}

\section{Introduction}

The exploration of the thermodynamic properties of binary polymer melts is of considerable interest in fundamental and applied research. ${ }^{1,2}$ On the fundamental side, the experimental technique of small-angle neutron scattering (SANS) plays a prominent role as it is a very sensitive and direct tool ; the scattering contrast between the two polymers can be made very large by the use of fully hydrogenated and deuterated components so that the scattering of neutrons from thermal composition fluctuations become strong and the dominant contribution. By the method of contrast variation cooperative phenomena as well as single-polymer properties can be studied in a blend. ${ }^{3,4}$

In this paper we present SANS scattering experiments on binary polymer blends to explore thermal composition fluctuation in different pressure and temperature fields. The basic samples are three binary polymer blends of nearly critical composition of polystyrene (PS) and three polybutadienes (PB) of different microstructure. The polybutadienes were fully deuterated (therefore d-PB) for reasons of strong scattering contrast. The d-PB polymers were synthesized by anionic polymerization from perdeuterated butadiene monomers. Three different solvent/cosolvent mixtures were used for the polymerization, leading to polymer chains with different ratios of 1,4 and 1,2 addition: d-PB $(1,4)$ with $7 \%$, d-PB $(1,2 ; 1,4)$ with $54 \%$, and $d-P B(1,2)$ with $91 \% 1,2$ (vinyl) repeat units. The d-PB polymers are effectively considered as "statistical copolymers" of 1,4 and 1,2 repeat units. The molar volumes of the polymers were about $2000 \mathrm{~cm}^{3} / \mathrm{mol}$. All samples show a phase boundary at temperatures between 60 and $90{ }^{\circ} \mathrm{C}$ and decompose into macroscopic large phases at low temperatures. The phase boundary is el evated by the vinyl

\footnotetext{
* To whom correspondence should be addressed.

† Institut für Festkörperforschung.

‡ Risø National Laboratory.
}

content of the PB chain, and accordingly different values for the Flory-Huggins $(\mathrm{FH})$ interaction parameters are evaluated from the SANS experiments. Because the phase boundary and the FH parameter of the d-PB/PS blend changes with vinyl content, a finite $\mathrm{FH}$ parameter must exist between the 1,4 and 1,2 repeat units. The theoretical approach for a blend consisting of statistical copolymers predicts a relatively strong repulsive intramolecular interaction between the 1,4 and 1,2 repeat units. However, an additional SANS experiment on a d-PB $(1,4) / h-P B(1,2)$ blend showed a much smaller FH parameter, in contradiction with the former experiments. The conclusion is that the existing theoretical description of statistical copolymers does not consistently describe our experimental results.

A further aspect of this work is related to the effect of thermal fluctuations on the thermodynamic properties as already discussed in earlier studies. ${ }^{5}$ The $\mathrm{FH}$ theory is based on a mean field approach, neglecting the effect of thermal composition fluctuations, and the FH parameter $\Gamma$ therefore represents a mean field or bare interaction parameter. From SANS experiments one usually gets $\Gamma=\Gamma_{\mathrm{h}} / T-\Gamma_{\sigma}$ with the corresponding phenomenological enthal pic and entropic terms $\Gamma_{\mathrm{h}}$ and $\Gamma_{\sigma}$, respectively. For symmetrical binary blends with polymer components of equal molar volume $\mathrm{V}$ the critical composition is $\Phi_{\mathrm{C}}=0.5$ and the $\mathrm{FH}$ parameter at the critical temperature $T_{C}$ is according to $T_{C}=2 N$ inversely proportional to the molar volume of the two components. However, the experiments are usually not performed in the range of mean field approximation. This is true for most binary polymer blends of upper critical solution type (UCST), whose molar volume has to be sufficiently small to show a moderate critical temperature. We therefore cannot directly analyze the experimental data with the $\mathrm{FH}$ model but need a more sophisticated model, which includes the effects of thermal fluctuations. We applied a crossover model derived by Belyakov et al.,, 6 which sufficiently describes well 
the scattering data, for example, the susceptibility, and derived the mean field parameters as the $\mathrm{FH}$ parameter from this model. ${ }^{5}$ This of course means that to get reliable fit parameters we have to study blends of very near critical composition, measure S(Q) over a sufficiently large range of temperature, and in very fine steps near the critical temperature.

A final aspect of this work is related to the effects of high external pressure fields on thermal fluctuations. Relevant insight can be obtained from the pressure dependence of the phase boundaries, the Ginzburg number, and the FH interaction parameter. The d-PB/ PS blends show a pressure-induced increase of the phase transition temperature and a decrease of the Ginzburg parameter and the FH parameter terms. The increase of the phase boundary with pressure is the normally expected case as it is usually related to a decrease of the free volume and the corresponding decrease of the entropic $\mathrm{FH}$ term $\Gamma_{\sigma}{ }^{8-10}$ According to the Clausius-Clapeyron equation, the pressure-induced changes of the phase boundary are determined by those of the FH parameter and of the Ginzburg number. ${ }^{11} \mathrm{~F}$ or the d-PB(1,2)/PS sample we find that the pressureinduced change of the critical temperature is dominated by the corresponding change of the Ginzburg number. The Clausius-Clapeyron equation leads to a consistency check of the experimental results and their underlying theoretical basis. Part of this work has already been published in ref 11.

\section{Theoretical Background}

In this section we will give a short review about the theoretical background of our experiments. The main part is related to the structure factor representing the experimental SANS results and is a measure of the thermal composition fluctuations from which the thermodynamic parameters were evaluated. The second part is related to expressions of the $\mathrm{FH}$ parameter for blends containing a statistical copolymer, and finally, the Clausius-Clapeyron equation is introduced.

A. Structure F actor and Susceptibility of Binary Polymer Blends. The scattering experiments were performed in the homogeneously mixed state of the samples at different temperature and pressure fields to measure the degree of thermal composition fluctuations from which equilibrium thermodynamic information is obtained. Thermal composition fluctuations lead to scattering of neutrons, which is expressed by the structure factor $S(Q)$ as a function of momentum transfer $\mathrm{Q}$. The value of $\mathrm{Q}$ is expressed by the scattering angle $\Theta$ and neutron wavelength $\lambda$ according to $Q=$ $(4 \pi / \lambda) \sin \Theta / 2$. At sufficiently small $Q$ values the structure factor can be approximated by the Zimm approximation ${ }^{3}$ according to

$$
\mathrm{S}^{-1}(\mathrm{Q})=\mathrm{S}^{-1}(0)+\mathrm{L}_{2} \mathrm{Q}^{2}
$$

The extrapolated $\mathrm{S}(0)$ represents a susceptibility and $L_{2}$ is related to the correlation length $\xi$ of the thermal fluctuations according to $L_{2}=\xi^{2} / S(0)$. Within the random phase approximation, 1,2 that is, within the mean field approximation, the radius of gyration $\mathrm{R}_{\mathrm{g}}$ and the statistical segment length $\sigma$ are obtained from $L_{2}$ according to the relations $\mathrm{L}_{2}=\mathrm{Rg}^{2} / 3 \mathrm{~S}(0 ; \Gamma=0)=$ $2 \mathrm{R}_{\mathrm{g}}{ }^{2} \Gamma_{\mathrm{S}} / 3=\mathrm{R}_{\mathrm{g}}{ }^{2} /[3 \Phi(1-\Phi) \mathrm{V}]$ and $\mathrm{R}_{\mathrm{g}}{ }^{2}=\sigma^{2} \mathrm{~N} / 6$ ( $\Gamma_{\mathrm{s}}$ is the $\mathrm{FH}$ parameter at spinodal; $\mathrm{N}$ is the degree of polymerization), respectively. The thermodynamic information is derived from the susceptibility $S(0)$, representing a property averaged over a macroscopic volume due to the inverse relationship between the reci procal momentum $\mathrm{Q}$ and real space. The susceptibility $\mathrm{S}(0)$ is related by the fluctuation-dissipation theorem with the second derivative of the Gibbs free energy of mixing with respect to composition according to $S^{-1}(0)=\partial^{2}\left(G_{m} / R T\right) /$ $\partial \Phi^{2}$ (R is the gas constant; $\Phi$ is the volume fraction of one component). ${ }^{12,13}$

The degree of thermal composition fluctuations and thereby S(Q) sensitively depends on temperature and pressure. At sufficiently high temperature above the critical temperature, the thermal fluctuations become weak so that they can be described within the Gaussian approximation of noninteracting fluctuation modes; in this state the system is well described by the mean field approximation. That is, most of the critical exponents are the same..$^{13}$ On the other hand, thermal fluctuations become very strong and interacting near the phase boundary and especially near the critical temperature, leading to nonlinear effects. These nonlinear effects become visible in scattering experiments by a crossover behavior of the susceptibility to the universality class of the three-dimensional (3D) I sing critical behavior, describing the fluctuations asymptotically close to the critical temperature.1,2 So asymptotically far and close to the critical temperature thermal composition fluctuations in polymer blends are described respectively within the universality classes of mean field and 3DI sing model. Within these two models the susceptibility $\mathrm{S}(0)$ is described by simple scaling laws according to $\mathrm{S}(0)$ $=\mathrm{C} \tau^{-\gamma}$ with the reduced temperature $\tau=\left|\mathrm{T}_{\mathrm{C}}-\mathrm{T}\right| / \mathrm{T}$ (critical temperature $\mathrm{T}_{\mathrm{C}}$ ) and with the critical exponent $\gamma$ being equal to 1 and 1.239 in the mean field and 3DI sing case, respectively. ${ }^{13}$ To describe the suscepti bility $\mathrm{S}(0)$ over the whole temperature range above the critical temperature, one needs a crossover function. We applied the asymptotic crossover model by Belyakov and Kisel ev ${ }^{6,7}$ according to

$$
\begin{aligned}
\hat{\tau}=\left(1+2.333 \hat{S}(0)^{\Delta / \gamma}\right)^{(\gamma-1) / \Delta} \times\left[\hat{S}^{-1}(0)+\right. & \left.\left(1+2.333 \hat{S}(0)^{\Delta / \gamma}\right)^{-\gamma / \Delta}\right]
\end{aligned}
$$

with the exponents $\gamma=1.234$ and $\Delta=0.5$ of the 3DIsing model; the rescal ed reduced temperature $\hat{\tau}=\tau / \mathrm{Gi}$ $\left(\tau=\left|T_{C}-\mathrm{T}\right| / T\right)$ is formulated as a function of the rescaled susceptibility $\hat{S}(0)=S(0) G i / C_{M F}$. The parameters $\mathrm{Gi}, \mathrm{C}_{\mathrm{MF}}$, and $\mathrm{T}_{\mathrm{C}}$ are the experimental results characterizing the system; $\mathrm{Gi}$ and $\mathrm{C}_{\mathrm{MF}}$ are the Ginzburg number and the mean field critical amplitude of $S(0)$, respectively. In the asymptotic limits $\tau \gg 1$ and $\tau \ll 1$ the susceptibility in eq 1 follows the well-known scaling laws $S(0)=C_{M F} \tau^{-1}$ of the mean field approximation and $\mathrm{S}(0)=\mathrm{C}_{+} \tau^{-\gamma}$ of the 3D-Ising model, respectively. This crossover function was already applied by us to other blend samples in earlier SANS work. ${ }^{5,11}$

The crossover between the two universality classes of mean field and 3D-I sing behavior is estimated by the Ginzburg criterion, delivering the Ginzburg number $\mathrm{Gi}$, which is a reduced crossover temperature and is related to the ratio of the critical amplitudes of the 3D-Ising and mean-field case according to $0^{5,7}$

$$
\mathrm{Gi}=0.069\left(\mathrm{C}_{+} / \mathrm{C}_{\mathrm{MF}}\right)^{1 /(\gamma-1)}
$$

with the 3D-I sing exponent $\gamma$. Within the $\mathrm{FH}$ model the susceptibility is given as $\mathrm{S}(0) N=\left[2\left(\Gamma_{\mathrm{C}} \mathrm{V}-\Gamma \mathrm{V}\right)\right]^{-1}$ with 


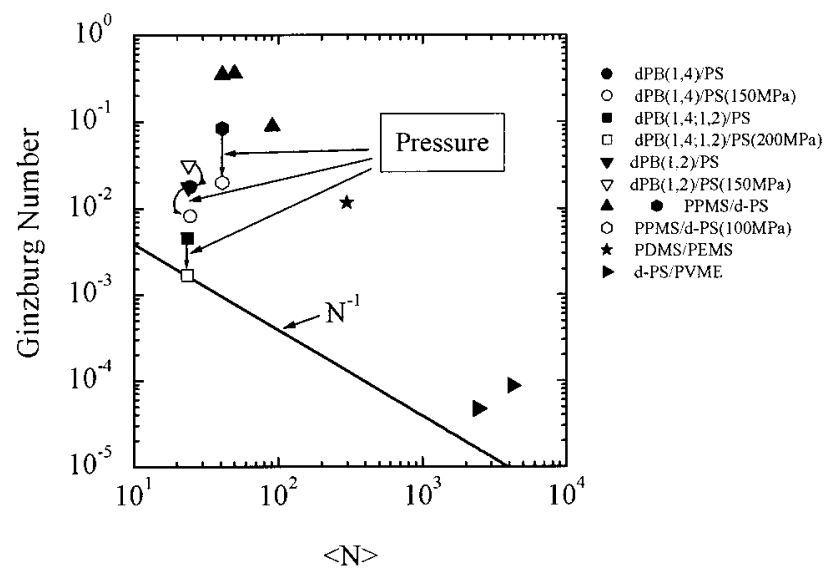

Figure 1. Ginzburg number $\mathrm{Gi}$ versus mean degree of polymerization for a collection of polymer blends. The values of $\mathrm{Gi}$ of the present blends at ambient- and high-pressure fields are shown (see legend at the figure).

the $\mathrm{FH}$ parameters $\Gamma$ and $\Gamma_{\mathrm{C}}$, representing $\Gamma$ at the critical temperature $T_{C}$. So the mean field critical amplitude is related to the $\mathrm{FH}$ parameters according to

$$
\mathrm{C}_{\mathrm{MF}}=1 / 2\left|\Gamma_{\mathrm{S}}+\Gamma_{\sigma}\right|=\mathrm{T}_{\mathrm{C}}^{\mathrm{MF}} / 2 \Gamma_{\mathrm{h}}
$$

with the corresponding enthal pic and entropic terms of $\Gamma$. The expressions of the critical amplitudes of the mean field case in eq 4 and of the 3D-I sing case according to $\mathrm{C}_{+} \propto \mathrm{V}^{(2-\gamma)}$ as derived by Binder ${ }^{14}$ leads to

$$
\mathrm{Gi} \propto\left[\mathrm{V}^{(2-\gamma)}\left(2 N+\Gamma_{\sigma}\right)\right]^{1 /(\gamma-1)}
$$

and which in the case of $\Gamma_{\sigma}=0$ becomes a universal scaling law according to $\mathrm{Gi} \propto 1 \mathrm{~N}$. The last relationship was originally derived by deGennes for incompressible polymer blends. ${ }^{1}$ So according to its dependence on molar volume, a very small $\mathrm{Gi}$ is expected in polymer blends if compared with a typical Gi in low molecular liquids, which is of the order of $10^{-2}{ }^{7}$ On the other hand, it becomes apparent from the large exponent $1 /(\gamma-1)$ $\cong 4.17$ in eq 5 that the entropic term $\Gamma_{\sigma}$ strongly enhances Gi. Such a behavior of Gi was indeed experimentally found demonstrated in Figure 1, showing a collection of Gi numbers from different polymer blends as determined by us over the recent years. All Gi values are appreciably larger (in some cases by 2 orders of magnitude and even larger than those for low molecular liquids) compared with the solid $1 / \mathrm{N}$ line for incompressible melts. These findings clearly indicate that the entropic term $\Gamma_{\sigma}$ (according to equation of states theories $\Gamma_{\sigma}$ is related to the compressibility ${ }^{2}$ ) leads to a strong increase of thermal composition fluctuations near the critical temperature. These large Gi numbers imply a need for a more sophisticated analysis of the scattering data, considering the effect of thermal fluctuations as has been done in the crossover function of eq 2. The F H parameter will therefore be evaluated from the mean field critical amplitude $\mathrm{C}_{\mathrm{MF}}$ of $\mathrm{S}(0)$ in eq 4 and from the "mean field" critical temperature $T_{C}^{\mathrm{MF}}$, which is related to the "real" critical temperature $\mathrm{T}_{\mathrm{C}}$ according to $\mathrm{T}_{\mathrm{C}}^{\mathrm{MF}}$ $=\mathrm{T}_{\mathrm{C}} /(1-\mathrm{Gi}) .{ }^{7} \mathrm{~T}_{\mathrm{C}}$ is smaller than $\mathrm{T}_{\mathrm{C}}^{\mathrm{MF}}$ by some degree Kelvin because of the stabilization effect of thermal fluctuations. In comparison with the mean field analysis the crossover function typically gives about 100\% and $50 \%$ larger entropic and enthal pic portions of the F lory-
Huggins parameter, respectively, for a $\mathrm{S}(0)$ with a Ginzburg number of the order of $10^{-2}$.

B. Clausius-Clapeyron Equation within the Flory-Huggins Model for Polymer Blends. The Clausius-Clapeyron equation describes the line of coexistence between two phases in the $T-P$ plane of the phase diagram according to

$$
\Delta \mathrm{T}_{\mathrm{C}} / \Delta \mathrm{P}=\mathrm{T}_{\mathrm{C}} \mathrm{V}_{\mathrm{m}} / \mathrm{H}_{\mathrm{m}}
$$

For example, the change of the phase transition (critical) temperature $T_{c}$ with pressure is related to the differences of volume $V_{m}$ and enthalpy $\mathrm{H}_{m}$ between the two phases. ${ }^{12} \mathrm{~F}$ or further analysis the Gibbs free energy of mixing is needed

$$
\mathrm{G}_{\mathrm{m}}(T, P, \Phi)=\mathrm{G}_{12}-\Phi \mathrm{G}_{1}+(1-\Phi) \mathrm{G}_{2}=\mathrm{H}_{\mathrm{m}}-\mathrm{TS}_{\mathrm{m}}
$$

with the symbols $\Phi$ representing the volume fraction of the component " 1 ", $G_{1}$ and $G_{2}$ the Gibbs potentials of the two components, and $\mathrm{G}_{1,2}$ that of the mixture. Within mean field theory the Flory-Huggins $(\mathrm{FH})$ model describes polymer blends; the enthalpy and entropy of mixing are given as

$$
\mathrm{H}_{\mathrm{m}} / \mathrm{RT}=\Phi(1-\Phi) \chi_{\mathrm{h}} / \mathrm{T}
$$

and

$$
\begin{array}{r}
\mathrm{S}_{\mathrm{m}} / \mathrm{R}=-\left\{\left(\Phi \mathrm{N}_{1}\right) \ln \Phi+\left[(1-\Phi) \mathrm{N}_{2}\right] \ln (1-\Phi)\right\}+ \\
\Phi(1-\Phi) \chi_{\sigma}
\end{array}
$$

with the molar vol umes $\mathrm{V}_{1}$ and $\mathrm{V}_{2}$ of the two molecular species. ${ }^{1,2}$ The first term of $\Delta \mathrm{S}_{\mathrm{m}}$ is the configurational part while the second one contains all the other entropic contributions as from polymer end effects and compressibility. ${ }^{2}$ The entropic term $\chi_{\sigma}$ is a phenomenological parameter that had to be included to describe the (mainly scattering) experiments. Usually, the enthal pic and entropic terms $\chi_{\mathrm{h}}$ and $\chi_{\sigma}$ are comprised within the $\mathrm{FH}$ interaction parameter according to $\chi=\chi_{\mathrm{h}} / \mathrm{T}-\chi_{\sigma}$.

The Gibbs free energy of mixing $G_{m}(T, P, \Phi)$ contains all thermodynamic information. It was already mentioned that the susceptibility $S(0)$ measured in SANS experiments is related to the second derivative of $\mathrm{G}_{\mathrm{m}^{-}}$ $(T, P, \Phi)$ with respect to composition. This also means that in scattering experiments one measures an effective $\mathrm{FH}$ parameter $\Gamma$ defined as $\partial^{2}[\phi(1-\phi) \chi] / \partial \phi^{2}$. The effective $\mathrm{FH}$ parameter $\Gamma$ is given in units $\left(\mathrm{mol} / \mathrm{cm}^{3}\right)$. The product of $(\Gamma \mathrm{V})$ has the same meaning as $(\chi N)$, sometimes given in corresponding SANS and theoretical papers. From now on we will denote $\Gamma$ as the $\mathrm{FH}$ parameter. In the case of a symmetrical blend with $\mathrm{V}$ $=\mathrm{V}_{1}=\mathrm{V}_{2}$ the critical composition is equal to $\Phi_{\mathrm{C}}=0.5$ with $\Gamma_{\mathrm{C}}=2 \mathrm{~N}$, which is the $\mathrm{FH}$ parameter at $\mathrm{T}_{\mathrm{C}}$.

The Clausius-Clapeyron equation for polymer blends can now be written as ${ }^{11}$

$$
\begin{aligned}
\partial \mathrm{T}_{\mathrm{C}} / \partial \mathrm{P}=\mathrm{T}_{\mathrm{C}}(1)\left[\partial \Gamma_{\mathrm{h}} / \partial \mathrm{P}-\mathrm{T}_{\mathrm{C}}(1) \partial \Gamma_{\sigma} / \partial \mathrm{P}\right] / \\
\Gamma_{\mathrm{h}}(1)-\left[\mathrm{T}_{\mathrm{C}}(1) /(1-\mathrm{Gi}(1))\right] \partial \mathrm{Gi} / \partial \mathrm{P}
\end{aligned}
$$

The first term has been derived from eqs 7-9 and $V_{m}$ $=\partial G_{m} / \partial P$. The second term describes the stabilization effect of thermal fluctuations described by the Ginzburg number $\mathrm{Gi}$ and the relationship between the critical temperature $\mathrm{T}_{\mathrm{C}}$ and its mean field value $\mathrm{T}_{\mathrm{C}}^{\mathrm{MF}}$ according 
Table 1. Polymer Chain Characteristics

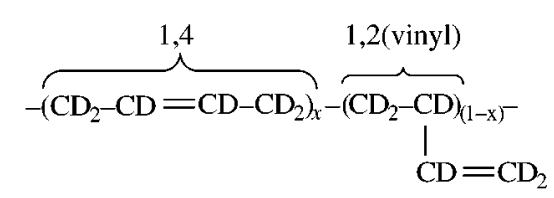

\begin{tabular}{|c|c|c|c|c|c|}
\hline \multirow{2}{*}{ polymer } & \multicolumn{4}{|c|}{ polybutadiene } & \multirow{2}{*}{$\frac{\text { polystyrene }}{\mathrm{PS}\left(\mathrm{C}_{8} \mathrm{H}_{8}\right)}$} \\
\hline & & $\mathrm{d}-\mathrm{PB}\left(\mathrm{C}_{4} \mathrm{D}_{6}\right)$ & & h-PB $\left(\mathrm{C}_{4} \mathrm{H}_{6}\right)$ & \\
\hline vinyl content (\%)a & $\begin{array}{l}7 \\
\text { d-PB }(1,4)\end{array}$ & $\begin{array}{c}54 \\
\text { d-PB }(1,2 ; 1,4)\end{array}$ & $\begin{array}{c}91 \\
\text { d-PB }(1,2)\end{array}$ & $\begin{array}{c}91 \\
\text { h-PB }(1,2)\end{array}$ & PS\#1 (PS\#2) \\
\hline$\Omega\left(\mathrm{cm}^{3} / \mathrm{mol}\right)^{\mathrm{b}}$ & 60.4 & & & & 99.1 \\
\hline$\rho\left(10^{10} \mathrm{~cm}^{-2}\right)^{\mathrm{c}}$ & 6.76 & & & 0.416 & 1.46 \\
\hline$M_{n} d$ & 2100 & 1900 & 2000 & 1900 & $1800(1700)$ \\
\hline $\mathrm{V}_{\mathrm{w}}\left(\mathrm{cm}^{3} / \mathrm{mol}\right)$ & 2200 & 2000 & 2100 & 2200 & 1800 (1700) \\
\hline$M_{w} / M_{n} e^{e}$ & 1.05 & 1.07 & 1.05 & 1.05 & 1.04 \\
\hline$\overline{\mathrm{N}}_{\mathrm{W}}$ & 36.4 & 33.1 & 34.8 & 36.4 & $18.2(17.2)$ \\
\hline
\end{tabular}

${ }^{a} \mathrm{By}{ }^{13} \mathrm{C} N M R$ in $\mathrm{CDCl}$ and ${ }^{1} \mathrm{H} N M R$ for $\mathrm{h}-\mathrm{PB}(1,2)$. ${ }^{\mathrm{b}}$ Monomer volume; densities: d-PB $=1 \mathrm{~g} / \mathrm{cm}^{3} ; \mathrm{h}-\mathrm{PB}=0.9 \mathrm{~g} / \mathrm{cm}^{3} ; \mathrm{h}-\mathrm{PS}=1.04 \mathrm{~g} / \mathrm{cm}^{3}$. ${ }^{c}$ Coherent scattering length density of the polymers determining the neutron scattering contrast. ${ }^{d}$ VPO (vapor pressure osmometry) at $\mathrm{T}=45^{\circ} \mathrm{C}$ in benzene. Their accuracy is within $\pm 5 \%$. ${ }^{\mathrm{e}} \mathrm{By} \mathrm{SEC}$ in THF.

to $\mathrm{T}_{\mathrm{C}}^{\mathrm{MF}}=\mathrm{T}_{\mathrm{C}} /(1-\mathrm{Gi}) .^{7}$ The parameters $\mathrm{T}_{\mathrm{C}}(1), \mathrm{Gi}(1)$, and $\Gamma(1)$ represent the corresponding values at the ambient pressure of $0.1 \mathrm{MPa}$.

C. Theory of Statistical Copolymers. According to a theoretical ansatz, the enthal pic FH parameter terms of a PS homopolymer and a $\mathrm{PB}(1,2 ; 1,4)$ statistical copolymer with vinyl content $\mathrm{n}$ is given as ${ }^{15,16}$

$$
\begin{array}{r}
\Gamma_{\mathrm{h}}(1,2 ; 1,4 / \mathrm{PS})=\varphi \Gamma_{\mathrm{h}}(1,2 / \mathrm{PS})+(1-\varphi) \Gamma_{\mathrm{h}}(1,4 / \mathrm{PS})- \\
\varphi(1-\varphi) \Gamma_{\mathrm{h}}(1,2 / 1,4)
\end{array}
$$

While the intermolecular interactions between the PS and the two 1,2 and 1,4 PB monomers superimpose linearly, the third term represents an intramolecular interaction between the 1,2 and 1,4 monomer units of the PB copolymer. A positive intramolecular interaction leads to a decrease of $\Gamma_{\mathrm{h}}$ and therefore to an enhanced compatibility of the mixture as often observed in polymer systems. ${ }^{16,17}$ The ansatz for the enthalpic $\mathrm{FH}$ parameter in eq 11 has been extended in recent lattice cluster theory (LCT) calculations and in addition an entropic term has been given. A simplified version of the FH parameter can be found in the "LCT for Pedestrian" by Dudowicz and Freed. ${ }^{18}$ Because the socalled "monomer site occupancy index" is the same for both d-PB units, their enthalpic expression in their eq 20 is the same as that given here in eq 11 . The entropic contribution is formulated as

$$
\Gamma_{\sigma}(1,2 ; 1,4 / \mathrm{PS})=-\sqrt{\left(\mathrm{S}_{\mathrm{PS}} \mathrm{S}_{\mathrm{PB}} / \Omega_{\mathrm{PS}} \Omega_{\mathrm{PB}}\right)}\left[\left(\mathrm{r}_{\mathrm{PS}}-\mathrm{r}_{\mathrm{PB}}\right)^{2} / \mathrm{z}^{2}\right]
$$

with $\mathrm{SPS}_{\mathrm{PS}}=8, \mathrm{SPB} 1,2_{\mathrm{P}}=\mathrm{S}_{\mathrm{PB} 1,4}=4, \mathrm{r}_{\mathrm{PS}}=1.375, \mathrm{r}_{\mathrm{PB}}=(\varphi+$ 4)/4, and the coordination number $z=6$. The monomer molar volumes are given in Table 1 . The entropic contribution according to the LCT calculation is al ways negative according to $\Gamma_{\sigma}(\mathrm{PB}(\varphi) / \mathrm{PS})=-1.27 \times 10^{-4}(1.5$ $-\varphi)^{2}$, delivering $\Gamma_{\sigma}(1,4 / \mathrm{PS})=-2.6 \times 10^{-4} \mathrm{~mol} / \mathrm{cm}^{3}, \Gamma_{\sigma^{-}}$ $(1,2 ; 1,4 / \mathrm{PS})=-1.2 \times 10^{-4} \mathrm{~mol} / \mathrm{cm}^{3}$, and $\Gamma_{\sigma}(1,2 / \mathrm{PS})=$ $-0.44 \times 10^{-4} \mathrm{~mol} / \mathrm{cm}^{3}$ for our samples.

D. Polydispersity of the Vinyl Content and Its Effect on the FH Parameter. A finite size distribution of polymers in blends leads to a distribution of the configurational entropy according to its proportionality with $1 / N$. Therefore, those systems represent a multicomponent system. Polydispersity of the polymer chain length sensitively influences the shape of the binodal

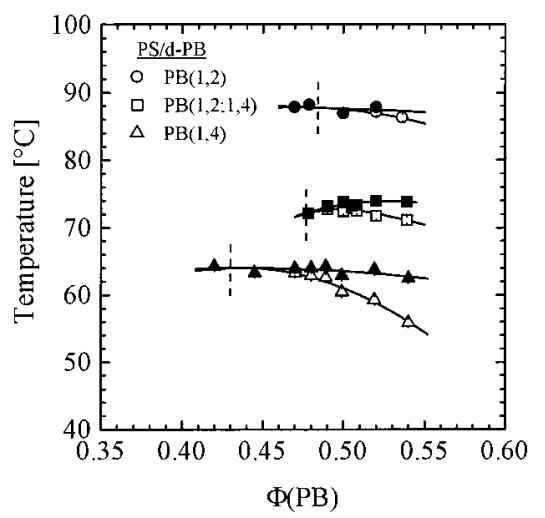

Figure 2. Phase diagram of the three d-PB/PS blends. The filled and open symbols correspond to the binodal and spinodal, respectively, and the dashed line locates the critical point.

while the spinodal is determined by the weight-averaged molar volumes. ${ }^{19}$ So in UCST (upper critical solution temperature) blends the critical temperature does not represent anymore the highest temperature of phase decomposition and the polydisperse chains are fractionated during phase separation. Those systems are also called "quasi" binary systems.

The phase diagram of the sample with the statistical d-PB $(1,2 ; 1,4)$ copolymer in Figure 2 shows phase boundaries that are typical for polydisperse systems as the critical temperature is not at the top of the binodal phase boundary. The presently studied polymers have low polydispersity with $\mathrm{M}_{\mathrm{w}} / \mathrm{M}_{\mathrm{n}}<1.1$ (see Table 1 ). However, the d-PB copolymer chains show statistical fluctuations of their vinyl content; the distribution of the vinyl content of the d-PB chains in a sample can be estimated on the basis of the binomial distribution; the 1,2 and 1,4 contents in the d-PB $(1,4)$ and d-PB $(1,2)$ are evaluated with an accuracy of about $5 \%$ while for the d-PB $(1,2 ; 1,4)$ of about $9 \%$. A distribution of the vinyl content becomes effective because of a finite $\mathrm{FH}$ parameter between the 1,2 and 1,4 units; a d-PB copolymer mixed with PS therefore represents a multicomponent system with respect to the FH parameter. For illustration a schematic phase diagram is shown in Figure 3 for constant temperature and pressure. The solid straight line in Figure 3 represents a binary blend of PS and d-PB with $50 \%$ vinyl content; a distribution of d-PB chains with different vinyl contents may lie within the two dotted lines. In those polydisperse systems the 


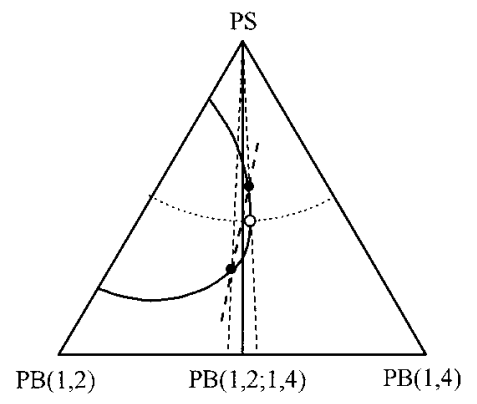

Figure 3. Schematic plot of a three-component blend to make plausible the observed characteristic features of binodal and spinodal of the d-PB $(1,2 ; 1,4) / P S$ blend in Figure 2 . I $n$ this blend the critical point is not found at the largest temperature of the phase boundary in contrast to the other two and which can be explained from polydispersity of the $\mathrm{FH}$ parameter.

composition of the phase-separated domains may be given by the full dots in Figure 3; this means that the d-PB component in the two domains will have different vinyl distributions. In case of such a fractionation the d-PB/PS blend can only be considered as a "quasi" binary system.

\section{Experimental Section}

The scattering experiments were performed with the SANS diffractometer KWS1 at the FRJ 2 reactor of the "Forschungszentrum J ülich". ${ }^{20} \mathrm{~F}$ or these studies a steel-bodied temperature-pressure cell was used, which allows an in situ change of pressure and temperature in the range of $0.1 \leq \mathrm{P}(\mathrm{MPa}) \leq$ 200 and $-20 \leq T\left({ }^{\circ} \mathrm{C}\right) \leq 200$, respectively, with a temperature control better than $0.01 \mathrm{~K}$. This cell was developed in our laboratory. The sample thickness and its neutron-irradiated diameter were 0.1 and $0.7 \mathrm{~cm}$, respectively. After each change of the temperature we waited for half an hour before proceeding with the neutron measurements to safely achieve a stationary temperature field in the relatively large volume of the pressure cell. The temperature of the sample could be rather accurately determined from the thermocouple and the always-existing temperature gradient between the sample and the position of the thermocouple. The temperature gradient was determined in a separate measurement under identical conditions with an additional thermocouple at the sample position. The scattering data were corrected for background, detection efficiency of the single-detector cells, radial averaged, and calibrated in absolute units by a Lupolen secondary standard. The resulting absolute macroscopic cross-section $\mathrm{d} \Sigma /$ $\mathrm{d} \Omega(\mathrm{Q})$ in units of $1 / \mathrm{cm}$ is related to the structure factor $\mathrm{S}(\mathrm{Q})$ according to $\mathrm{d} \Sigma / \mathrm{d} \Omega(\mathrm{Q})=\mathrm{S}(\mathrm{Q}) \Delta \rho^{2} / \mathrm{N}_{\mathrm{A}}$, where $\Delta \rho$ is the difference of the coherent scattering length densities of the polymer components $\Sigma c_{\mathrm{i}} b_{\mathrm{i}} / \Omega_{\mathrm{i}}$ (numerical values are given in Table 1 ) and the Avogadro number $\mathrm{N}_{\mathrm{A}}$. The scattering experiments were performed in the momentum transfer range of $0.01 \leq$ $Q\left(\AA^{-1}\right) \leq 0.1$ using the settings of 7- $\AA$ neutron wavel ength and the detector-to-sample distances of 4 and $8 \mathrm{~m}$ with the corresponding collimations distances.

The polymers were synthesized by anionic polymerization using high vacuum and break seal technique. sec-Butyllithium was used as the initiator for all polymerizations. The purification of solvents and monomers was performed according to the standards required for anionic polymerization. ${ }^{21}$ Protonated polystyrene (PS) and deuterated polybutadiene (d-PB) with a 1,4 microstructure were polymerized in benzene. The preparation of polybutadienes with an increased 1,2 content demands the addition of polar cosolvents. Tetrahydrofuran (THF) was the cosolvent for polymers with high vinyl content (h-,d-PB$(1,2))$ while di ethyl ether was used for the polybutadiene with al most equal distribution of 1,4 and 1,2 units in the chain (d$\mathrm{PB}(1,2 ; 1,4))$.

The polydispersity, $M_{w} / M_{n}$, of the polymers was determined by size exdusion chromatography using a Waters $150 \mathrm{C}$ instrument. This instrument was equipped with four $\mu$-Styragel
Table 2. Critical Point of Polymer Blends with PS\#2 Component According to the Results in Figure 2

\begin{tabular}{lcccc} 
sample & d-PB $(1,4) / P S$ & $d-P B(1,2 ; 1,4) / P S$ & d-PB $(1,2) / P S$ & $d-P B(1,4) / P B(1,2)$ \\
\hline$\Phi_{c}(P B)$ & 0.43 & 0.477 & 0.484 & 0.5
\end{tabular}

$\begin{array}{lllll}\mathrm{T}_{\mathrm{C}}\left({ }^{\circ} \mathrm{C}\right) & 64 & 72.2 & 8.48 & 0.5 \\ & & & 88\end{array}$

columns with a porosity range of $10^{5}-500 \AA$ and one ultraStyragel column of continuous porosity in combination with a refractive index detector. The eluent was THF at a flow rate of $1 \mathrm{~mL} / \mathrm{min}$ and PS standards (Tosoh Corporation) were used for calibration. In all cases $M_{w} / M_{n}$ values were smaller than 1.1. The number-averaged molecular weight of the polymers, $M_{n}$, was measured with a Knauer vapor pressure osmometer at $45^{\circ} \mathrm{C}$ in benzene. The $M_{n}$ values were obtained using a calibration function of the type $(\Delta \mathrm{V} / \mathrm{c})_{\mathrm{c} \rightarrow 0}=\mathrm{KM}_{n}^{\alpha}$, where $\Delta \mathrm{V}$ is the change of the thermistors in voltage and $\mathrm{c}$ is the concentration. ${ }^{22}$ The calibration constants $K$ and $\alpha$ were determined with benzene and PS standards of $440 \leq M_{n} \leq 9600$ with an accuracy of about $\pm 5 \%$. The $1,2 / 1,4$ composition of the d-polybutadienes was determined by ${ }^{13} \mathrm{C}$ NMR spectroscopy with a Bruker 300-M Hz spectrometer. For the h-PB $(1,2)$ polymer the composition was additionally determined by ${ }^{1} \mathrm{H}$ NMR. The characteristic parameters of the polymers are summarized in Table 1.

The polymer blends were prepared by dissolving in and freeze-drying from benzene. The thermal stability of the samples was improved by the addition of $0.1 \% 2,6$ di-tert-butyl4-methylphenol and by filling them in an argon box into a leakproofed sample holder. In this way the blends were protected from degradation and cross-linking at the experimental temperatures.

To measure precisely the effect of thermal fluctuations and their pressure dependence, we had to prepare samples very near the critical composition. In a first step we therefore determined the binodal and spinodal phase boundaries of the samples at different compositions by SANS measurements. The so-obtained phase diagram of the three samples is plotted in Figure 2 and the corresponding critical composition and temperature are given in Table 2. For these samples a PS component (PS\#2) of $\mathrm{V}_{\mathrm{w}}=1700 \mathrm{~cm}^{3} / \mathrm{mol}$ molar volume was used, which is slightly smaller than the PS component (PS\#1) $V_{w}=$ $1800 \mathrm{~cm}^{3} / \mathrm{mol}$ mainly used later in the pressure experiments (Table 1) and is the reason of slightly smaller phase boundary temperatures. The critical temperatures given in Figure 2 are different by $22 \mathrm{~K}$ and are increasing with the vinyl content of the d-PB polymer. The chosen composition of the three samples for the pressure experiments are given in Table 3. The composition for the samples used in pressure experiments were chosen to be the critical ones within the accuracy of determination. For the d-PB(1,2;1,4)/PS blend the first phase boundary experiments gave misleadingly a critical composition of 0.505 , which had to be corrected afterward.

\section{Experimental Results}

In this section we first present the SANS data and then will give an interpretation in terms of the crossover function, the theory for statistical copolymers, and the Clausius-Clapeyron equation.

A. SANS Data. Structure factors $S(Q)$ have been plotted in Zimm representation in Figure 4, namely, the inverse $\mathrm{S}(\mathrm{Q})$ versus the momentun transfer $\mathrm{Q}^{2}$. The experimental data follow in all cases a straight line in accordance with the Zimm approximation (eq 1). F rom the fit of the straight line the susceptibility $\mathrm{S}(0)$ and from the slope the coefficient $L_{2}$ is eval uated. In Figure 4a the temperature was changed in a constant pressure field while in Figure $4 \mathrm{~b}$ the pressure was changed at constant temperature. Decreasing the temperature leads to a strong enhancement of $\mathrm{S}(\mathrm{Q})$ as one approaches the critical temperature and thereby to stronger thermal fluctuations. A similar increase of $\mathrm{S}(\mathrm{Q})$ is observed at constant temperature by increasing the 
Table 3. Experimental Results of the Blends from Pressure Experiments ${ }^{a}$

\begin{tabular}{|c|c|c|c|c|}
\hline \multirow[b]{2}{*}{ sample } & \multicolumn{2}{|c|}{ d-PB $(1,4) / P S \# 2$} & \multicolumn{2}{|c|}{ d-PB $(1,2 ; 1,4) / P S \# 1$} \\
\hline & $X(P=0.1 \mathrm{MPa})$ & $\partial \mathrm{X} / \partial \mathrm{P}(* / \mathrm{MPa})$ & $\mathrm{X}(\mathrm{P}=0.1 \mathrm{MPa})$ & $\partial \mathrm{X} / \partial \mathrm{P}(* / \mathrm{MPa})$ \\
\hline \multirow[t]{3}{*}{$\begin{array}{l}\Phi(\mathrm{d}-\mathrm{PB}) \\
\overline{\mathrm{N}}_{\mathrm{W}} \\
\Gamma_{\mathrm{S}}\left(10^{-3} \mathrm{~mol} / \mathrm{cm}^{3}\right) \\
\mathrm{T}_{\mathrm{B}}\left({ }^{\circ} \mathrm{C}\right) \\
\mathrm{T}_{\mathrm{S} ; \mathrm{C}}\left({ }^{\circ} \mathrm{C}\right) \\
\text { Ginzburg number } \\
\mathrm{C}_{\mathrm{MF}}\left(\mathrm{cm}^{3} / \mathrm{mol}\right) \\
\mathrm{C}_{+}\left(\mathrm{cm}^{3} / \mathrm{mol}\right) \\
\Gamma_{\mathrm{h}}\left(\mathrm{mol}^{-3} / \mathrm{cm}^{3}\right) \\
\Gamma_{\sigma}\left(10^{-3} \mathrm{~mol} / \mathrm{cm}^{3}\right)\end{array}$} & $\begin{array}{l}0.42 \\
24.8 \\
1.05 \\
65.2 \pm 0.1 \\
63.4 \pm 0.1 \\
(1.8 \pm 0.2) \times 10^{-2} \\
152 \pm 1.1 \\
111 \pm 1.3 \\
1.12 \pm 0.02 \\
2.24 \pm 0.05\end{array}$ & $\begin{array}{l}(7.6 \pm 0.1) \times 10^{-2} \\
(7.7 \pm 0.1) \times 10^{-2} \\
-(6.4 \pm 1) \times 10^{-5} \\
(1.65 \pm 0.04) \times 10^{-1} \\
-(2.7 \pm 0.1) \times 10^{-2} \\
-(8.9 \pm 0.2) \times 10^{-4} \\
-(3.1 \pm 0.2) \times 10^{-3}\end{array}$ & $\begin{array}{l}0.505 \\
23.4 \\
1.06 \\
85.5 \pm 0.2 \\
82.6 \pm 0.1 \\
(0.45 \pm 0.15) \times 10^{-2} \\
181 \pm 3 \\
92 \pm 3 \\
0.99 \pm 0.02 \\
1.7 \pm 0.05\end{array}$ & $\begin{array}{l}(6.64 \pm 0.1) \times 10^{-2} \\
(6.6 \pm 0.1) \times 10^{-2} \\
-(2.3 \pm 1.1) \times 10^{-5} \\
(4.2 \pm 0.2) \times 10^{-1} \\
(4.5 \pm 0.3) \times 10^{-2} \\
-(1.6 \pm 0.08) \times 10^{-3} \\
-(4.8 \pm 0.3) \times 10^{-3}\end{array}$ \\
\hline & & \multicolumn{3}{|c|}{ d-PB $(1,2) / P S \# 1$} \\
\hline & sample & $\mathrm{X}(\mathrm{P}=0.1 \mathrm{MPa})$ & \multicolumn{2}{|c|}{$\partial \mathrm{X} / \partial \mathrm{P}(* / \mathrm{MPa})$} \\
\hline $\begin{array}{l}\Phi(\mathrm{d}-\mathrm{PB}) \\
\overline{\mathrm{N}}_{\mathrm{W}} \\
\Gamma_{\mathrm{S}}\left(10^{-3}\right. \\
\mathrm{T}_{\mathrm{B}}\left({ }^{\circ} \mathrm{C}\right) \\
\mathrm{T}_{\mathrm{S} ; \mathrm{C}}\left({ }^{\circ} \mathrm{C}\right) \\
\mathrm{Ginzbur} \\
\mathrm{C}_{\mathrm{MF}}\left(\mathrm{cm}^{3}\right. \\
\mathrm{C}_{+}\left(\mathrm{cm}^{3} /\right. \\
\Gamma_{\mathrm{h}}\left(\mathrm{mol} \mathrm{K}^{\mathrm{K}}\right. \\
\Gamma_{\sigma}\left(10^{-3} \mathrm{r}\right.\end{array}$ & & $\begin{array}{l}0.5 \\
23.9 \\
1.03 \\
101 \pm 0.6 \\
101 \pm 0.5 \\
(3.2 \pm 0.3) \times 10^{-2} \\
134 \pm 2 \\
112 \pm 4 \\
1.44 \pm 0.03 \\
2.7 \pm 0.05\end{array}$ & \multicolumn{2}{|c|}{$\begin{array}{l}(2.5 \pm 0.1) \times 10^{-2} \\
(2.6 \pm 0.1) \times 10^{-2} \\
-(0.94 \pm 0.1) \times 10^{-4} \\
(1.6 \pm 0.04) \times 10^{-1} \\
(1.7 \pm 0.09) \times 10^{-2} \\
-(1.5 \pm 0.05) \times 10^{-3} \\
-(3.7 \pm 0.1) \times 10^{-3}\end{array}$} \\
\hline
\end{tabular}

a The given error bars in all Tables were obtained from the statistical methods of the fitting routines including the statistical errors of neutron data.

pressure field from 0.1 to $150 \mathrm{MPa}$; the phase boundaries were enhanced by pressure. In Figure 4c S(Q) of a PB mixture is shown with components of the d-PB$(1,4)$ and h-PB $(1,2)$ microstructure (see Table 1$)$. The scattering of this mixture is appreciably weaker with a slightly enhanced scattering at $10{ }^{\circ} \mathrm{C}$ with respect to $153^{\circ} \mathrm{C}$. The phase boundary of the latter sample is expected at around $-210^{\circ} \mathrm{C}$, as estimated from extrapolation of the corresponding susceptibilities in Figure 13.

In Figure $5 a-c$ the inverse susceptibility of all investigated d-PB/PS samples and all pressure fields are plotted versus inverse temperature. These measured $\mathrm{S}(0)$ values were fitted by the crossover function (eq 2) depicted as solid lines. The agreement of the fitted lines with the corresponding experimental $\mathrm{S}(0)$ is very good. From these fits the spinodal or critical temperature at $\mathrm{S}^{-1}(0)=0$, the critical mean field amplitude $\mathrm{C}_{\mathrm{MF}}$ and the Ginzburg number Gi were obtained and are summarized in Table 3. In F igure 6 the inverse susceptibility of the two blends d-PB $(1,4 ; 1,2) / P S$ and $d-P B(1,4) / P S$ have been plotted; their measurements were repeated at $0.1 \mathrm{MPa}$ in our standard heater instead of the pressure cell for reasons that will become clear below. The corresponding fit parameters are given in Table 4.

B. Analysis of the SANS Data. In Figure 7 the spinodal (critical) and binodal temperatures of the three samples have been depicted versus pressure. The spinodal temperature is obtained from the extrapolated $\mathrm{S}^{-1}(0)=0$ and the binodal is determined from a sudden decrease of $\mathrm{S}(0)$, which occurs after passing the binodal line; bel ow the binodal one measures thermal fluctuations within the precipitated domains, which strongly decrease with decreasing temperature. The corresponding $S^{-1}(0)$ have not been pl otted in Figure 5 but similar observations have been discussed in ref 23 . In all samples an increase of the phase boundaries with pressure is observed. The shape of the phase boundary is linear for the blends with the d-PB $(1,4)$ and $d-P B-$ $(1,4 ; 1,2)$ copolymer and it is more parabolic for the blend with the d-PB $(1,2)$. The critical composition was very well found for the d-PB $(1,2)$ sample while the other two samples are slightly off the critical composition as seen from the distances of the spinodal and binodal temperatures. The worst compatibility is observed for the d-PB$(1,2) / P S$ sample, the best one for the $d-P B(1,4) / P S$, and for the copolymer sample in between.

The Ginzburg numbers are depicted in Figure 8 versus pressure. The fitted lines are a guide for the eye. Appreciable differences are observed; the largest $\mathrm{Gi}$ is found for the d-PB $(1,2)$ blend while the lowest one for the copolymer blend and an intermediate $\mathrm{Gi}$ for the d-PB $(1,4)$ blend. In all cases $G$ i decreases with increasing pressure; for the d-PB $(1,4)$ blend one observes a linear decrease, for the d-PB $(1,2)$ a constant $\mathrm{Gi}$ up to $100 \mathrm{M} \mathrm{Pa}$ and then a rather strong decrease, and for the copolymer blend a decrease at low pressure and a constant Gi at larger pressure, suggesting a saturated value of $\mathrm{Gi}=(1.7 \pm 0.5) \times 10^{-3}$. This value seems to be very near the Gi estimated originally for incompressible polymer blends (see Figure 1).

In Figure 9 the critical amplitudes of the susceptibility have been plotted versus pressure. The mean field critical amplitude $\mathrm{C}_{\mathrm{MF}}$ is a fit parameter of the crossover function. The critical amplitude $\mathrm{C}_{+}$of the 3D-Ising model has been evaluated from $\mathrm{Gi}$ and $\mathrm{C}_{\mathrm{MF}}$ (eq 3 ). Within an accuracy of $3 \%$ and $30 \%$ the $C_{+}$critical amplitude is independent of pressure and of microstructural effects, respectively. The $\mathrm{C}_{\mathrm{MF}}$ critical amplitudes increase with pressure and the largest value is observed for the copolymer blend.

From the mean field critical amplitude $\mathrm{C}_{\mathrm{MF}}$ the $\mathrm{FH}$ parameter was evaluated. According to eq 4, the entropic term $\Gamma_{\sigma}$ is directly obtained from $\mathrm{C}_{\mathrm{MF}}$ and $\Gamma_{\mathrm{C}}$ while the calculation of the enthalpic term $\Gamma_{\mathrm{h}}$ needs the mean field critical temperature according to $\mathrm{T}_{\mathrm{C}}^{\mathrm{MF}}=\mathrm{T}_{\mathrm{C}} /(1-$ $\mathrm{Gi})$. The enthalpic and entropic terms of the $\mathrm{FH}$ parameter are plotted in Figure 10 versus pressure. In all cases slightly declining values are observed with pressure. The terms of the $\mathrm{FH}$ parameter are largest for the d-PB $(1,2)$ and smallest for the copolymer. While 
(a)

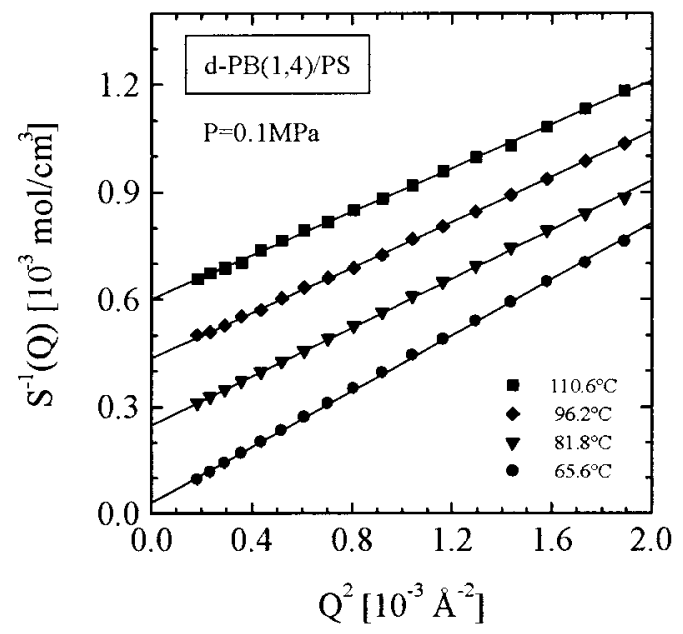

(c)

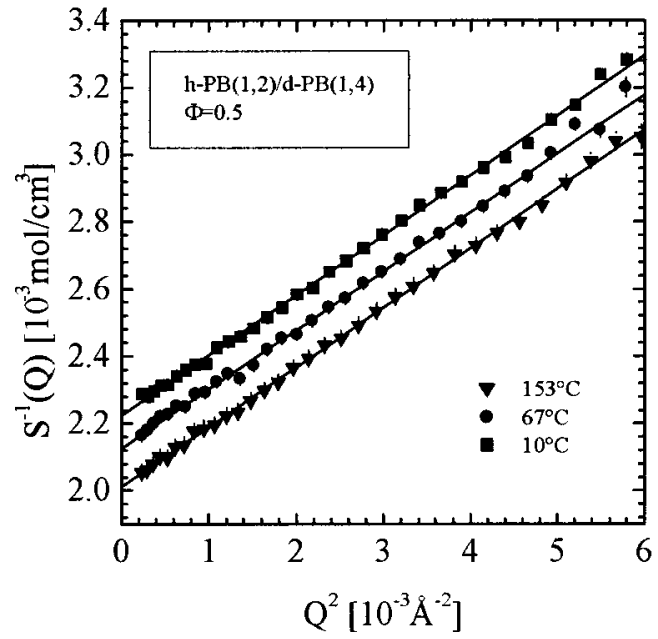

(b)

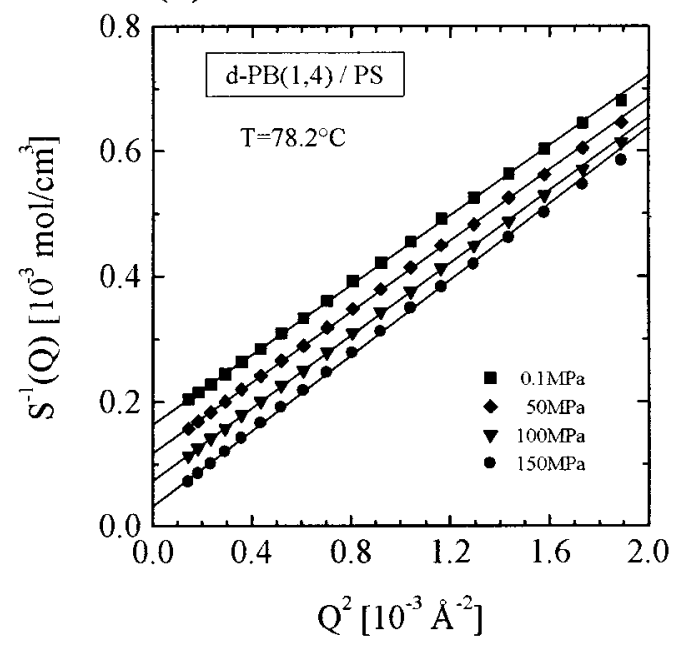

Figure 4. Structure factor in Zimm representation for the d-PB(1,4)/PS blend at constant pressure (a) and temperature (b) and for varying temperature and pressure fields, respectively. The third plot (c) shows the structure factor of the $h-\operatorname{PB}(1,2) / d-P B(1,4)$ blend; its temperature dependence comes mainly from a finite $\mathrm{FH}$ parameter between the $\mathrm{PB}(1,2)$ and $\mathrm{PB}(1,4)$ units.

the pressure dependence is linear for the $d-P B(1,4)$ blends, it is different for the other two samples; at ambient pressure the d-PB $(1,2)$ blend shows nearly no change of the $\mathrm{FH}$ parameter while in this pressure range it is rather strong for the copolymer blend becoming constant above $100 \mathrm{MPa}$.

C. Interpretation in Terms of the Theory of Copolymers. The enthal pic and entropic terms of the $\mathrm{FH}$ parameter in Figure 10 have been plotted in Figure 11 versus the vinyl content and fitted with the theoretical ansatz of statistical copolymers (eq 11). These fits are shown by the solid lines. In Figure 12 both corresponding terms of the $\mathrm{FH}$ parameter of the 1,4 and 1,2 butadiene repeat units with PS and with each other are depicted versus pressure. Equation 11 was originally derived for the enthalpic term; we applied the same ansatz also for the entropic term. The relatively small terms of the $\mathrm{FH}$ parameter obtained for the copolymer sample (see Figure 11) are interpreted by relatively strong terms of the intramolecular $\mathrm{FH}$ parameter between the 1,4 and 1,2 units. As shown in Figure 12, these values are even larger than the corresponding intermolecular interaction terms between the d-PB and PS units. This result is a rather surprising finding. It is known from literature that a finite intramolecular interaction leads to an improved compatibility of the blends. Such an improved compatibilty between PS and the d-PB $(1,2 ; 1,4)$ polymers, however, was not observed, as seen from the phase diagrams in Figures 2 and 7; the phase boundaries of the copolymer blend is between the two other ones. The phase boundaries are of course consistent with the evaluated $\mathrm{FH}$ parameter; according to eq 4 the mean field spinodal temperature is needed for the evaluation of the enthalpic term of the $\mathrm{FH}$ parameter.

In a subsequent SANS experiment the $\mathrm{FH}$ parameter between the 1,2 and 1,4 repeat units was determined directly from the h-PB $(1,2) / d-P B(1,4)$ mixture whose parameters are given in Tables 1 and 2 . The corresponding inverse susceptibility has been plotted versus the inverse temperature in Figure 13 . The $L_{2}$ values not shown here are constant within the error bars and deliver a mean statistical segment length of $\sigma=6.9 \AA$ from the random phase approximation according to $L_{2}$ $=2 \mathrm{R}_{\mathrm{g}}{ }^{2} \Gamma_{\mathrm{C}} / 3, \mathrm{R}_{\mathrm{g}}{ }^{2}=\sigma^{2} \mathrm{~V} /(6 \Omega)$, and $\Gamma_{\mathrm{C}}=2 \mathrm{~N}$. The corre sponding $\mathrm{FH}$ parameter has been evaluated from the susceptibility according to the mean field approximation $\mathrm{S}(0) N=\left[2\left(\Gamma_{\mathrm{C}} \mathrm{V}-\Gamma \mathrm{V}\right)\right]^{-1}$ and plotted in Figure 13. The observed enthal pic and entropic terms of the $\mathrm{FH}$ parameter are of the order of magnitude smaller than the values in Figure 12 prediced by the copolymer theory. The accuracy of the entropic term is mainly determined 
(a)

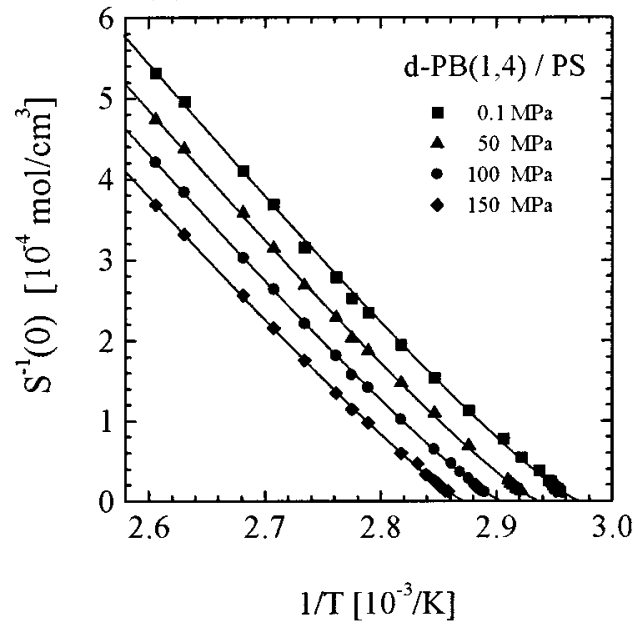

(c)

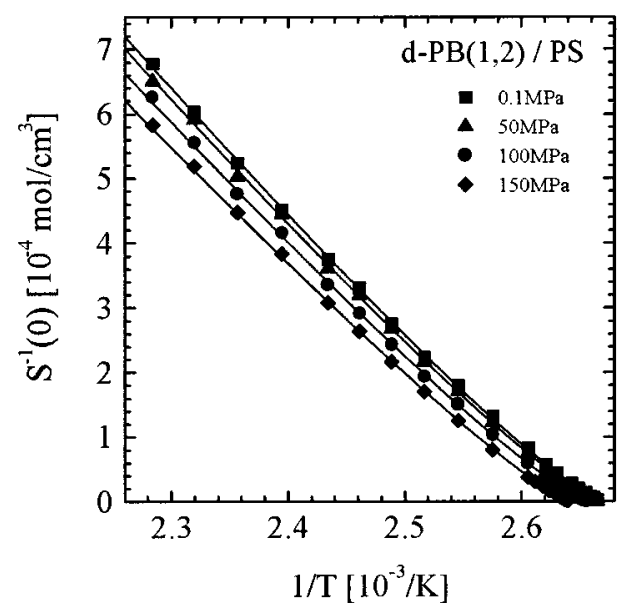

(b)

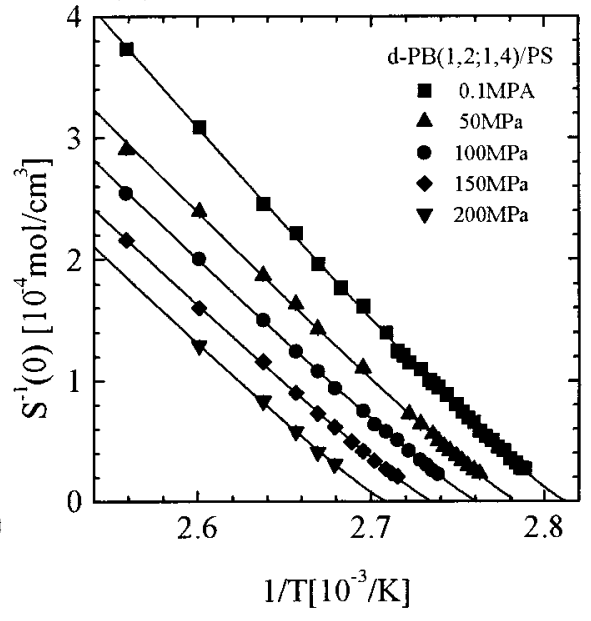

Figure 5. Inverse susceptibility versus inverse temperature for the three investigated d-PB/PS blends for the pressure fields studied. The solid lines represent the fit with the crossover function formulated in eq 2. Parameters are given in Table 3.

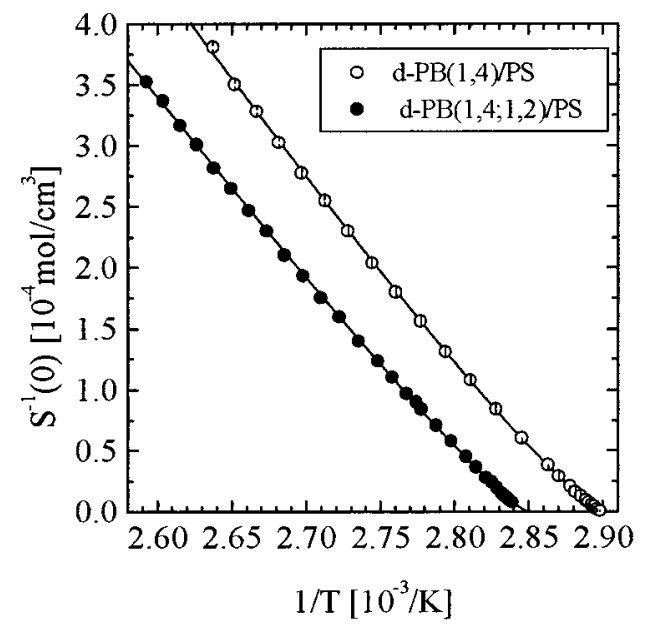

Figure 6. Inverse susceptibility versus inverse temperature of two blends with $(1,4)$ and the statistical copolymer $(1,4 ; 1,2)$ d-PB component measured at ambient pressure with our standard heater. Parameters are given in Table 4.

by $\Gamma_{C}$, which can be determined roughly within $\pm 5 \%$ according to the accuracy of the molar volume. As $\Gamma_{\sigma}$ in Table 5 is about half as large as $\Gamma_{C}$, its value is reliable within $\pm 10 \%$.

In Figure 14 the $\mathrm{FH}$ parameter of the blends of $\mathrm{d}-\mathrm{PB}$ $(1,4)$, d-PB $(1,2 ; 1,4)$, and $d-P B(1,2)$ components mixed
Table 4. Results of Two d-PB/PS Blends with PS\#1 as the Component Measured with the Heater

\begin{tabular}{|c|c|c|}
\hline sample & $\mathrm{d}-\mathrm{PB}(1,4) / \mathrm{PS}$ & d-PB $(1,4 ; 1,2) / P S$ \\
\hline$\Phi(d-P B)$ & 0.42 & 0.505 \\
\hline$\overline{\mathrm{N}}_{\mathrm{W}}$ & 25.6 & 23.4 \\
\hline$\Gamma_{\mathrm{C}}\left(10^{-3} \mathrm{~mol} / \mathrm{cm}^{3}\right)$ & 1.02 & 1.06 \\
\hline $\mathrm{T}_{\mathrm{C}}\left({ }^{\circ} \mathrm{C}\right)$ & $71.8 \pm 0.1$ & $78.1 \pm 0.1$ \\
\hline Ginzburg number $\left(10^{-2}\right)$ & $1.56 \pm 0.25$ & $0.46 \pm 0.06$ \\
\hline $\mathrm{C}_{\mathrm{MF}}\left(\mathrm{cm}^{3} / \mathrm{mol}\right)$ & $156 \pm 3.7$ & $193 \pm 3$ \\
\hline $\mathrm{C}_{+}\left(\mathrm{cm}^{3} / \mathrm{mol}\right)$ & $110 \pm 2$ & $101 \pm 2$ \\
\hline$\Gamma_{\mathrm{h}}\left(\mathrm{mol} \mathrm{K} / \mathrm{cm}^{3}\right)$ & $1.12 \pm 0.03$ & $0.91 \pm 0.02$ \\
\hline$\Gamma_{\sigma}\left(10^{-3} \mathrm{~mol} / \mathrm{cm}^{3}\right)$ & $2.19 \pm 0.08$ & $1.53 \pm 0.04$ \\
\hline
\end{tabular}

with PS have been plotted versus $1 / T$. Their phase transition temperatures were determined by the intercepts with $\Gamma_{C}$ as plotted. In addition, $\Gamma$ of the h-PB $(1,2) /$ d-PB $(1,4)$ from Figure 13 has been plotted. We find a strong discrepancy of the enthal pic and entropic terms of the $\mathrm{FH}$ parameter between the 1,4 and 1,2 units of the PB components, depending on whether they were determined directly from intermol ecular interactions of the d-PB $(1,4) / P B(1,2)$ blend or from intramolecular interactions of the d-PB $(1,2 ; 1,4)$ statistical copolymer mixed with the PS polymer, applying the theoretical approach in eq 11.

The experiments with our pressure were partly reconsidered with our standard heating system on the d-PB $(1,4) / P S$ and the copolymer d-PB $(1,4 ; 1,2) / P S$ blends 


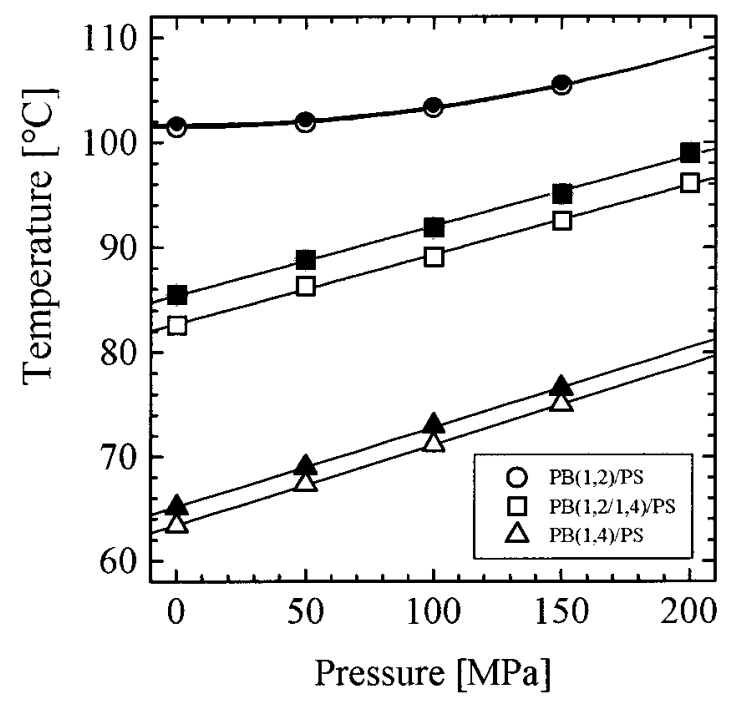

Figure 7. Spinodal and binodal versus pressure of the three blends.

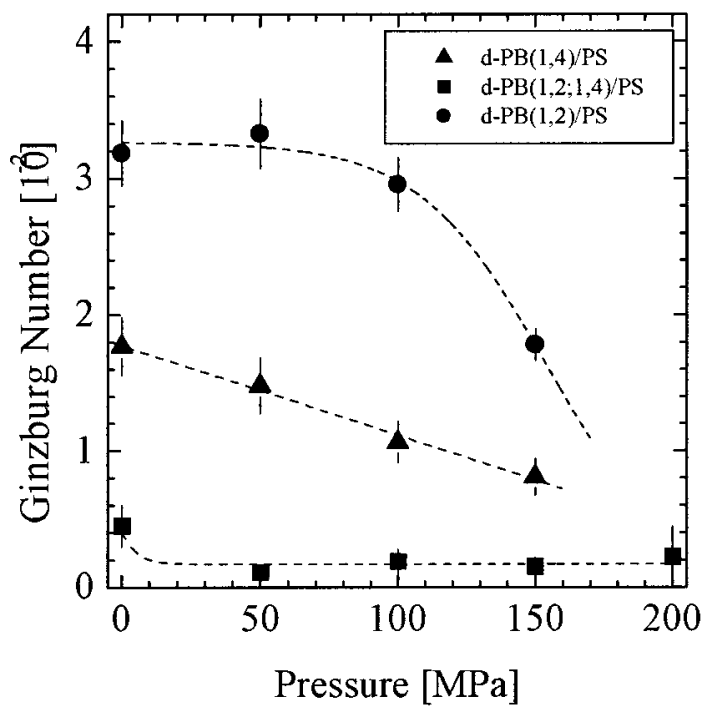

Figure 8. Ginzburg number Gi versus pressure of the three blends.

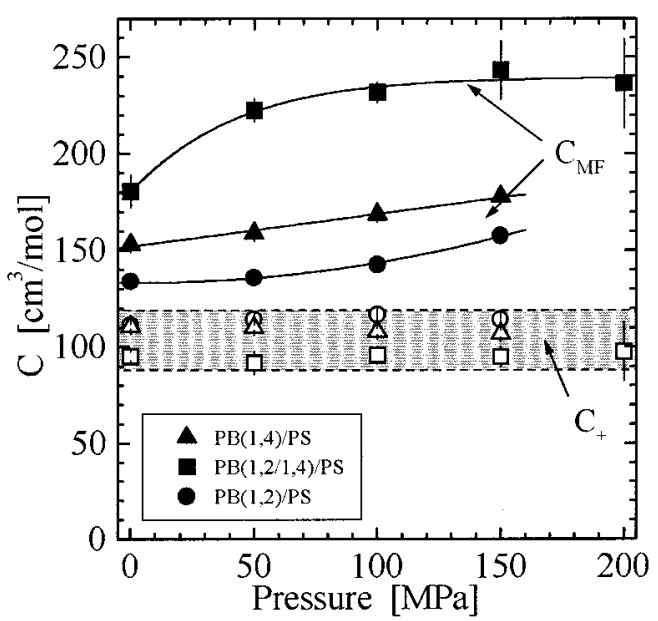

Figure 9. Critical amplitudes of mean field $\left(\mathrm{C}_{\mathrm{MF}}\right)$ and 3DIsing $\left(\mathrm{C}_{+}\right)$susceptibility versus pressure. $\mathrm{C}_{+}$is constant within $3 \%$ versus pressure and $30 \%$ versus the vinyl content.

to cross-check the experimental procedure. In Figure 6 the susceptibility and the fitted crossover functions (eq 2) have been plotted versus temperature. The evaluated

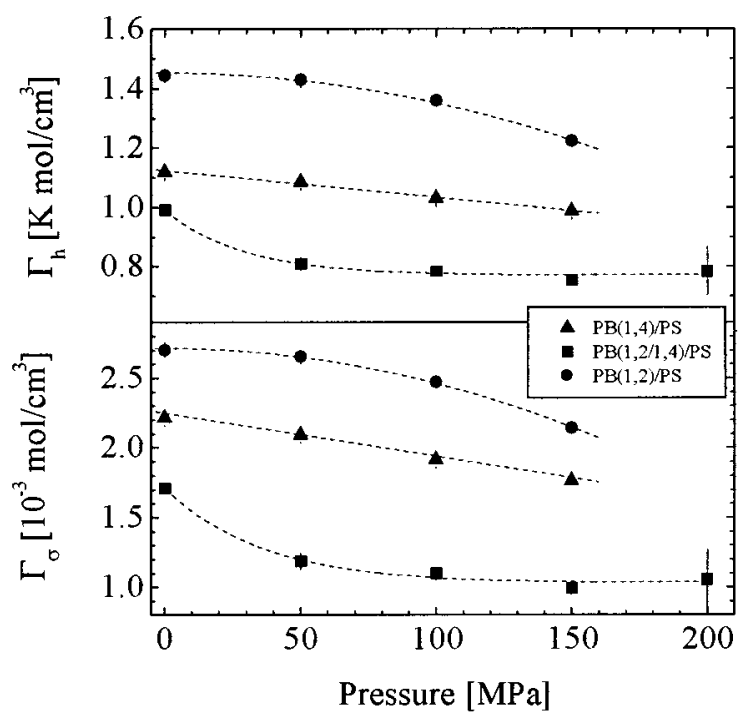

Figure 10. Enthalpic and entropic terms of the $\mathrm{FH}$ parameter versus pressure for the three d-PB/PS blends. The parameter is largest for the d-PB $(1,4)$ and smallest for the statistical copolymer blend.

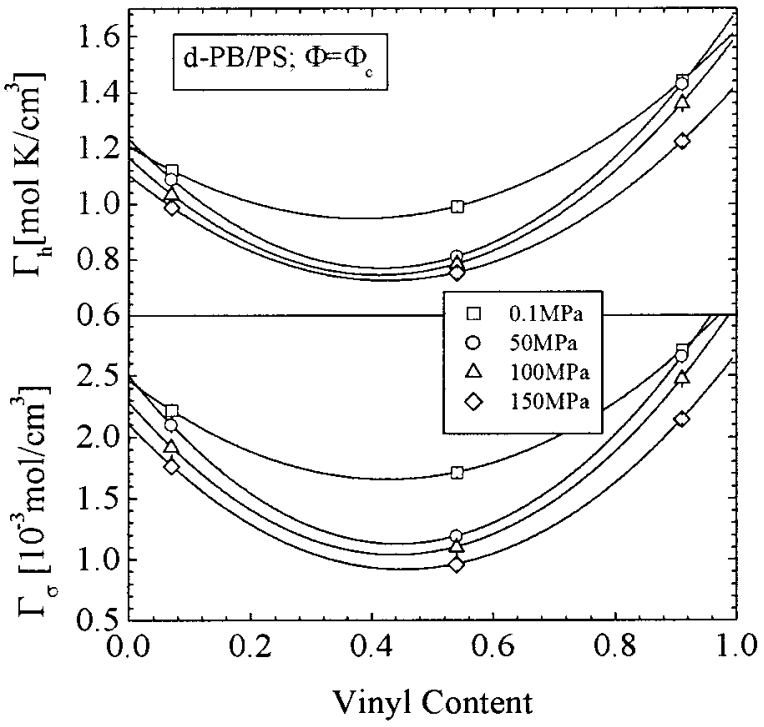

Figure 11. Enthalpic and entropic terms of the $\mathrm{FH}$ parameter versus vinyl content and for the pressure fields. The solid lines represent fits with the ansatz for statistical copolymers as given in eq 11.

results are summarized in Table 4; they are consistent with the corresponding parameters in Table 2. So from the experimental side we can safely conclude that the statistical copolymer theory in eq 11 leads to inconsistent interpretations.

While the enthalpic and entropic $\mathrm{FH}$ parameter portions of the $\mathrm{PB}(1,4) / \mathrm{PB} 1,2)$ blend obtained from the $\mathrm{d}-\mathrm{PB} / \mathrm{PS}$ blend are large in comparison with the corresponding intermolecular values (Figure 12), the $\mathrm{FH}$ parameter itself is rather small near the critical temperatures of the d-PB/PS blends (Figure 14). The reason is the large entropic contribution according to the observed negligible compatibilization effect of the copolymer mixture. This also means that an entropic term of the $\mathrm{FH}$ parameter has to be considered in contrast to the presently available theoretical description of copolymers. ${ }^{17,18}$

D. Application of Clausius-Clapeyron Equation. In Figure 15 the pressure-induced changes of the phase 


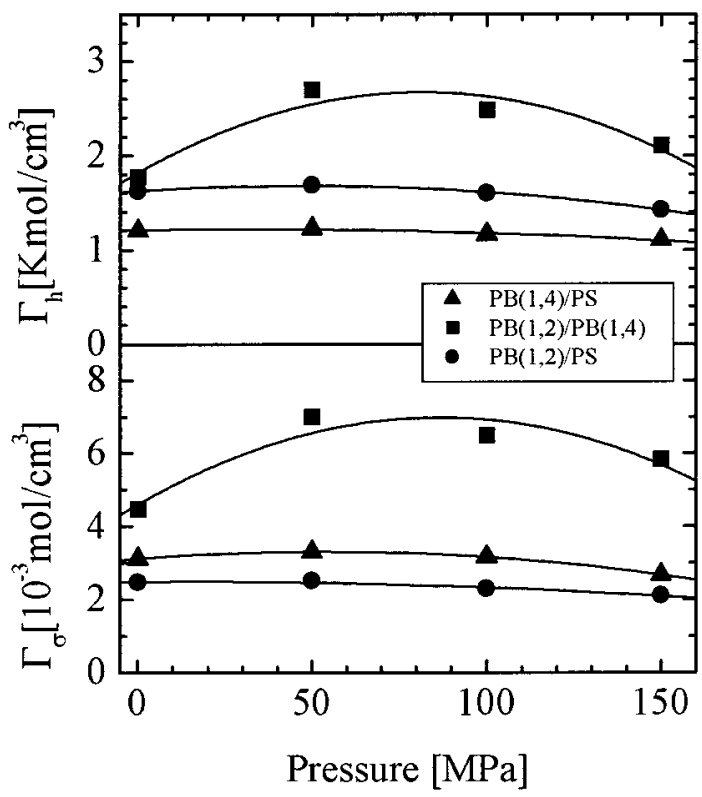

Figure 12. Parameters from the fit in Figure 11 versus pressure. The enthal pic and entropic terms are largest for the $\mathrm{PB}(1,2)$ and $\mathrm{PB}(1,4)$ representing an intramolecular interaction.
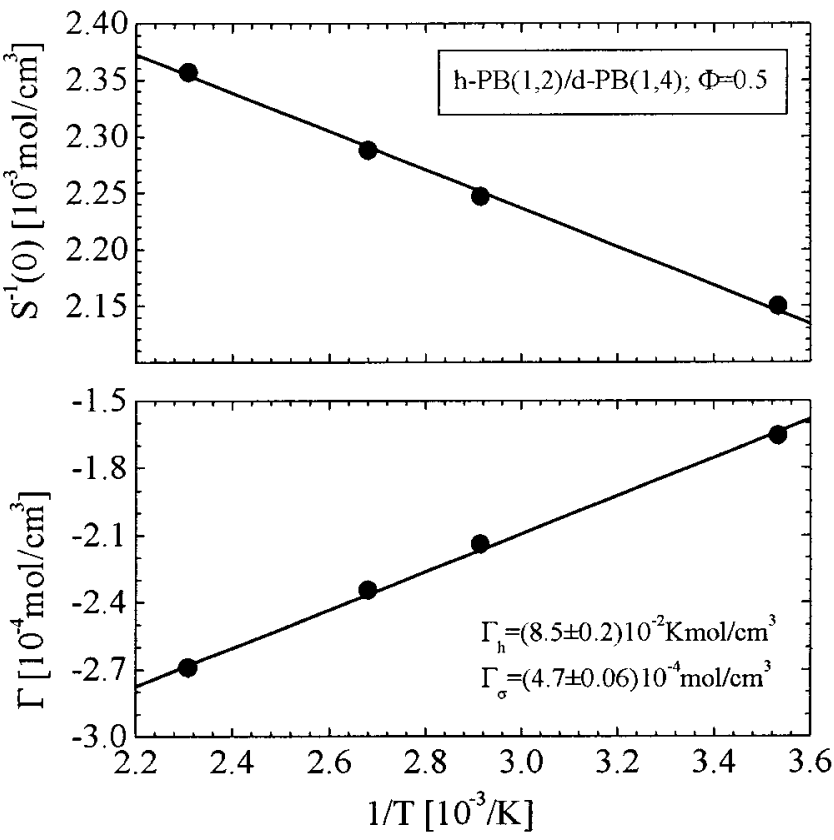

Figure 13. Inverse susceptibility and $\mathrm{FH}$ parameter versus inverse temperature of $(1,2)$ and $(1,4)$ PB blends. The FH parameter represents an intermolecular interaction. These values are 30-50 times smaller than the corresponding intramolecular values given in Figure 13.

Table 5. Results of the h-PB $(1,2) / d-P B(1,4)$ Blend

\begin{tabular}{ll}
\hline \multicolumn{1}{c}{ sample } & h-PB $(1,2) / \mathrm{d}-\mathrm{PB}(1,4)$ \\
\hline$\Phi$ & 0.5 \\
segment length $\sigma(\AA)$ & $6.92 \pm 0.05$ \\
$\Gamma_{\mathrm{C}}\left(10^{-4} \mathrm{~mol} / \mathrm{cm}^{3}\right)\left(\overline{\mathrm{N}}_{\mathrm{W}}\right)$ & $9.09(36.4)$ \\
$\mathrm{T}_{\mathrm{C}}\left({ }^{\circ} \mathrm{C}\right)$ & $\approx-210$ \\
$\Gamma_{\mathrm{h}}\left(10^{-2} \mathrm{~mol} \mathrm{~K} / \mathrm{cm}^{3}\right)$ & $8.5 \pm 0.2$ \\
$\Gamma_{\sigma}\left(10^{-4} \mathrm{~mol} / \mathrm{cm}^{3}\right)$ & $4.7 \pm 0.06$
\end{tabular}

boundary temperatures have been depicted versus the vinyl content. The derivative of temperature with respect to pressure was always averaged over the pressure range between 0.1 and $150 \mathrm{MPa}$. The full dots show $\Delta \mathrm{T} c / \Delta \mathrm{P}$ directly derived from the temperature of

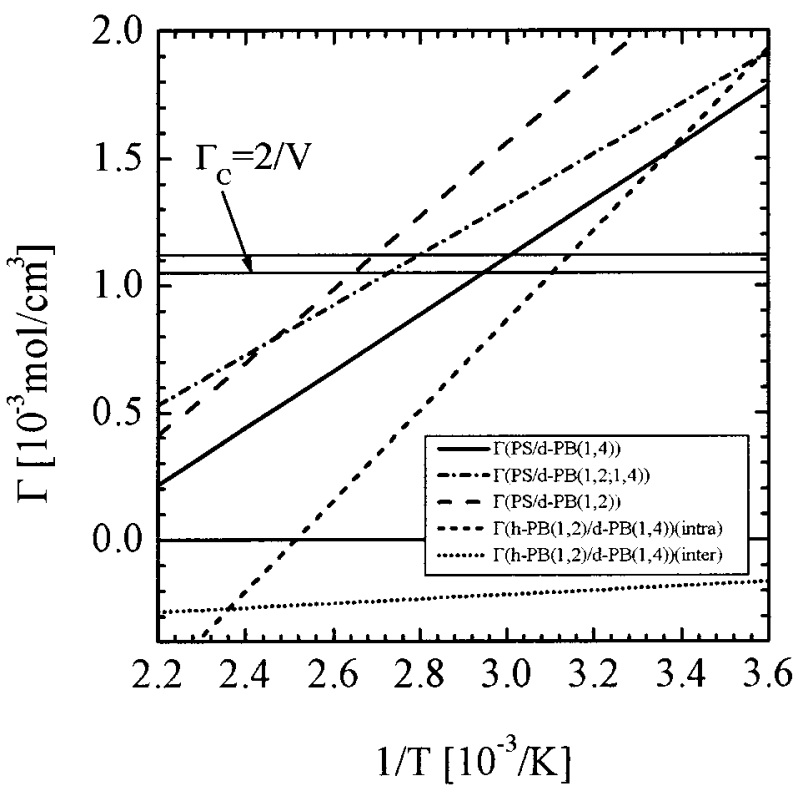

Figure 14. $\mathrm{FH}$ parameter at ambient pressure versus inverse temperature for the three d-PB/PS blends together with interand intramolecular $\mathrm{FH}$ parameter of the h-PB $(1,2) / d-P B(1,4)$ blend. In the ranges of the critical temperatures of the PB/PS blends the $\mathrm{FH}$ parameters of the $\mathrm{PB}(1,2) / \mathrm{PB}(1,4)$ are small and therefore no improved compatibility can be expected. If only the enthal pic term would be important as suggested from the theory of copolymers, then such an effect should occur in contrast to observation.

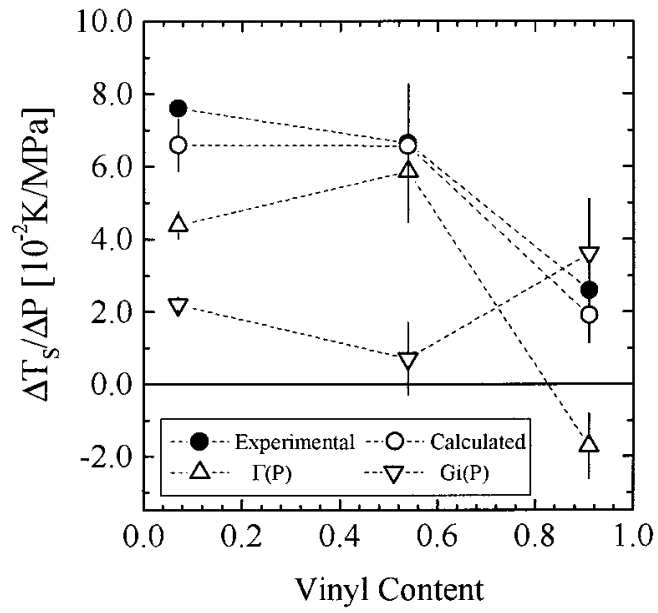

Figure 15. Change of spinodal (critical) temperature with pressure averaged over a pressure range of $150 \mathrm{MPa}$ obtained from experiment (full dots) and from Clausius-Clapeyron equation (open dots). In all cases a positive change is observed, which is largest for $(1,4)$ and smallest for the $(1,2)$ blend. There are two contributions, namely, from pressure-induced changes of the Ginzburg number and the $\mathrm{FH}$ parameter, as indicated by the triangles. In the $(1,2)$ blend the $\mathrm{FH}$ parameter causes a negative change of the spinodal temperature according to the data in Table 6.

the phase boundary while the open symbols represent the $\Delta T_{C} / \Delta P$ evaluated from the Clausius-Clapeyron equation in eq 10. The two triangular symbols show changes of temperature caused by the pressure-induced changes of the $\mathrm{FH}$ parameter and by the corresponding changes of the Ginzburg number; the open dots repre sent the sum of both contributions. For the two samples with the smaller vinyl content the effect from the $\mathrm{FH}$ parameter is about twice as large as those from the Ginzburg number $\mathrm{Gi}$, while for the d-PB(1,2)/PS sample a negative $\Delta T_{S} / \Delta \mathrm{P}$ is obtained from $\Gamma(\mathrm{P})$, which is more 
than compensated by $\mathrm{Gi}(\mathrm{P})$, giving a resulting positive $\Delta T_{C} / \Delta P$. The values of $\Delta T_{s} / \Delta P$ evaluated from the Clausius-Clapeyron equation are in good agreement with the experimental values.

\section{Summary and Discussion}

In this paper we present SANS experiments on three d-PB/PS polymer blends of nearly critical composition and molar volumes of about $2000 \mathrm{~cm}^{3} / \mathrm{mol}$. The three samples differ by the vinyl content of the d-PB component being $7 \%, 54 \%$, and $91 \%$ and thereby approximately representing $\mathrm{d}-\mathrm{PB}(1,4), \mathrm{d}-\mathrm{PB}(1,2 ; 1,4)$, and d-PB$(1,2)$ polymer chains. The experiments were performed at external fields of pressure between 0.1 and $200 \mathrm{MPa}$ and of temperature within the disordered regime from high to low temperatures until the direct neighborhood of the critical point. In SANS experiments the susceptibility $S(0)$ is determined from extrapolation of the structure factor $\mathrm{S}(\mathrm{Q})$ at $\mathrm{Q}=0 ; \mathrm{S}(0)$ is the basic experimental parameter and is a measure of the thermal composition fluctuations from which according to the fluctuation-dissipation theorem all the other thermodynamic information is derived.

The basic part of this paper is related to the observation that thermal composition fluctuations are too strong over the investigated temperature range to be described within the mean field approximation. This means that the Flory-Huggins $(\mathrm{FH})$ theory in general cannot be applied to the data analysis. Instead, we used a crossover function for $S(0)$ that describes the observed crossover behavior between mean field and 3D-Ising behaviors well. From the fit of the crossover function one gets three parameters, namely, the Ginzburg number Gi, the critical mean field amplitude of the susceptibility $\mathrm{C}_{\mathrm{MF}}$, and the critical temperature $\mathrm{T}_{\mathrm{c}}$. From these parameters one evaluates the $\mathrm{FH}$ interaction parameter and the critical amplitude $C_{+}$of the susceptibility within the 3D-Ising region. The values for $\mathrm{C}_{+}$are found constant within $30 \%$ for all three d-PB/PS samples and for all pressure fields. The phase boundaries of the three samples are different; the critical temperature continuously increases with increasing vinyl content of the d-PB component from about 65 to $100{ }^{\circ} \mathrm{C}$.

An external pressure field leads to an increase of the critical temperature with a rate between $8 \times 10^{-2}$ and $3 \times 10^{-2} \mathrm{~K} / \mathrm{MPa}$; the lowest rate is observed for the sample with the highest vinyl content. The Ginzburg number and the $\mathrm{FH}$ parameter of the three samples react differently on pressure: $\mathrm{Gi}$ is largest for the d-PB$(1,2)$ and strongly decreases above $100 \mathrm{MPa}$, while it is smallest for the d-PB $(1,2 ; 1,4)$ copolymer, which above $20 \mathrm{MPa}$ al ready approaches a constant value. This low Gi seems to correspond to the incompressible blend. The Gi for the d-PB $(1,4)$ blend is intermediate between the two other samples and linearly decreases with pressure. A pressure-induced decrease of $\mathrm{Gi}$ is expected from a decrease of the entropic term $\Gamma_{\sigma}$ (eq 5) and its relationship to compressibility predicted by equation of states theories. ${ }^{2}$ The critical amplitude $\mathrm{C}_{\mathrm{MF}}$ is another fit parameter of the crossover function (Figure 8); from $\mathrm{Gi}$ and $\mathrm{C}_{\mathrm{MF}}$, the critical amplitude $\mathrm{C}_{+}$is derived as shown in Figure 9. From $C_{M F}$ and the critical temperature one evaluates the $\mathrm{FH}$ parameter as depicted in Figure 10; the d-PB $(1,4)$ sample shows the largest values while the d-PB $(1,2 ; 1,4)$ copolymer sample the lowest ones, and in all samples the enthalpic and entropic terms decrease with pressure.
Table 6. Changes of Enthalpic and Entropic FH Parameter with Pressure Leading to $\Delta T_{B} / \Delta P$ as Plotted in Figure 15

\begin{tabular}{lcccc}
\hline & $\Gamma_{\mathrm{h}} / \Gamma_{\mathrm{B}}$ & $\partial \Gamma_{\mathrm{h}} / \partial \mathrm{P}$ & $\mathrm{T}_{\mathrm{B}} \partial \Gamma_{\sigma} / \partial \mathrm{P}$ & $\Delta \mathrm{T}_{\mathrm{B}} / \Delta \mathrm{P}$ \\
\hline $\mathrm{d}-\mathrm{PB}(1,4) / \mathrm{PS}$ & $>0$ & $-(9 \pm 0.2) \times 10^{-4}$ & $-(10.5 \pm 0.06) \times 10^{-4}$ & $>0$ \\
d-PB $(1,2 ; 1,4) / \mathrm{PS}$ & $>0$ & $-(16 \pm 0.8) \times 10^{-4}$ & $-(17 \pm 1) \times 10^{-4}$ & $>0$ \\
d-PB(1,2)/PS & $>0$ & $-(15 \pm 0.5) \times 10^{-4}$ & $-(13.8 \pm 0.4) \times 10^{-4}$ & $<0$
\end{tabular}

According to the Clausius-Clapeyron equation in eq 10 , the pressure-induced changes of the critical temperature are interrelated by those of the Ginzburg number and the $\mathrm{FH}$ parameter. The al ways observed pressure-induced decrease of $\mathrm{Gi}(\mathrm{P})$ leads to an increase of the critical temperature because of the stabilization effect of thermal fluctuations; the corresponding change of temperature has been depicted in Figure 15. The effects from pressure-induced changes of $\Gamma(P)$ are slightly more complicated as seen from eq 10. As the enthal pic term is al ways positive and both $\mathrm{FH}$ parameter terms decrease with pressure (see values in Table 6), one expects at low temperatures always a negative change of critical temperature with pressure. From the values in Table 6 one derives temperatures of 14,60, and 132 ${ }^{\circ} \mathrm{C}$ for the d-PB $(1,4)$, d-PB $(1,2 ; 1,4)$, and d-PB(1,2) samples, respectively, below which a negative $\Delta T_{B} / \Delta P$ is expected. So for the first two mentioned samples one evaluates a positive change of temperature in agreement with the experimental findings, as the corresponding critical temperatures are larger than 14 and $60^{\circ} \mathrm{C}$. For the d-PB $(1,2)$ sample, however, the critical temperature of $101{ }^{\circ} \mathrm{C}$ is smaller than the calculated $132{ }^{\circ} \mathrm{C}$ and therefore a negative change of critical temperature with pressure is evaluated from the corresponding change of $\Gamma(P)$ (see Figure 15). The sum from the contributions of $\Gamma(P)$ and $\mathrm{Gi}(\mathrm{P})$ yield values depicted as open dots in Figure 15 and are compared with the actually measured ones plotted as full dots. The agreement between both numbers is very good; they are within the experimental accuracy the same. This shows that the underlying theory of the crossover function (eq 2) used for the analysis of the susceptibility and the subsequent evaluation of the $\mathrm{FH}$ parameter and Ginzburg number leads to consistent results. It, furthermore, shows that pressure-induced changes of the phase boundaries are generally influenced by two effects, namely, by changes of the interaction parameter and by changes of the degree of thermal composition fluctuations.

The Ginzburg number of the three d-PB/PS samples and their changes with pressure have been plotted in Figure 1 versus the mean degree of polymerization and are compared with other $\mathrm{Gi}$ numbers. At ambient pressure one generally finds Ginzburg numbers that are larger and sometimes even of the order of magnitude larger than estimated by deGennes' universal $\mathrm{N}^{-1}$ scaling. So Gi of the d-PB(1,4)/PS and the d-PB $(1,2) / P S$ blends are consistently found to be 10-15 times larger than estimated. On the other hand, the PB $(1,2 ; 1,4) / P S$ copolymer blend shows only a 2.5 times larger Ginzburg number. So it can be concluded from the different Gi's of the three d-PB/PS blends with approximately the same molar volume that the Ginzburg criterion of polymer blends is not described by a universal function of the degree of polymerization or molar volume. As motivated by eq 5 and also shown by former pressure experiments $^{10}$ (see also PPMS/d-PS in Figure 1), the Ginzburg number sensitively depends on the entropic term of the $\mathrm{FH}$ parameter $\Gamma_{\sigma}$. This finding is clearly 
confirmed by our work. The pressure dependence of the Ginzburg number (Figure 8) is consistently much stronger than the corresponding pressure dependence of $\Gamma_{\sigma}$ (Figure 10) for all vinyl contents because of the large exponent in eq 5 . The values of $\mathrm{Gi}$ and $\Gamma_{\sigma}$ depend on the vinyl content in the same manner (Figures 8 and 10) because of the constant critical amplitude $C_{+}$(Figure 9), which is within 3\% and $30 \%$ independent of pressure and of vinyl content, respectively. The Ginzburg number and $\Gamma_{\sigma}$ of the d-PB $(1,2 ; 1,4) / P S$ blend approach constant values at pressures of larger than $50 \mathrm{MPa}$; at these pressure fields $\Gamma_{\sigma}$ and $\mathrm{Gi}$ seem to be near their incompressible values. Thus, our detailed experiments confirm that the Ginzburg number sensitively depends on the entropic contribution $\Gamma_{\sigma}$, which is the reason that (i) it is a nonuniversal quantity and (ii) it sensitively depends on external pressure fields and on polymeric microstructure. Only at sufficiently high pressure fields Gi seems to approach a value estimated originally by deGennes. ${ }^{1}$

Another aspect of this work is related to the observed $\mathrm{FH}$ parameter of the three samples and its interpretation within the theory of copolymers given in eq 11. Its analysis leads to surprisingly large intramolecular enthal pic and entropic $\mathrm{FH}$ parameter terms between the $\mathrm{PB}(1,4)$ and $\mathrm{PB}(1,2)$ units as depicted in Figure 12 and which are even larger than the corresponding intermolecular interaction terms between the PS and d-PB $(1,4)$ and $\mathrm{d}-\mathrm{PB}(1,2)$ units. These experimental findings were confirmed from additional SANS measurements of the d-PB $(1,4) / P S$ and the d-PB $(1,2 ; 1,4) / P S$ blends with our standard heater system. The corresponding results in Figure 6 and in Table 4 are consistent with those obtained from the pressure cell (see Table 3). The large intramolecular $\mathrm{FH}$ parameters evaluated for the PB$(1,4) / \mathrm{PB}(1,2)$ units from the theoretical approach for statistical copolymers in eq 11 were later tested on a h-PB $(1,2) / d-P B(1,4)$ mixture by additional SANS experiments. The corresponding intermolecular terms of the $\mathrm{FH}$ parameter given in Figure 14 are of the order of magnitude smaller and therefore in disagreement with the findings from the d-PB/PS blends. This means that the theory of copolymers as given in eq 11 does not lead to consistent results, at least for the presently investigated system. On the other hand, the $\mathrm{FH}$ parameter itself is very small in the temperature range of the critical temperatures because of the large entropic contribution (Figure 14).

There are only a few SANS experiments that study the effect of intramolecular interaction in polymer blends with statistical copolymer components and no experiments are known to us where intra- and intermolecular interactions have been studied simultaneously. Up to now the theoretical approach for statistical copolymers in eq 11 was mainly tested from determination of the phase boundary; the observation of an improved compatibility of statistical copolymers was then interpreted by a positive intramolecular enthalpic interaction. ${ }^{15-17}$ Those experiments cannot distinguish whether the $\mathrm{FH}$ parameter is a purely enthalpic or a a free energy parameter. In the SANS investigations of the d-PB/PS blends presented here we find a large positive intramolecular enthalpic $\mathrm{FH}$ parameter between the 1,4 and 1,2 d-PB units, even though we did not find any improved compatibility between the two components (see Figures 2 and 7). The reason is the large entopic contribution of the $\mathrm{FH}$ parameter that makes the "total" intramolecular FH parameter relatively small in the temperature range of phase separation as depicted in Figure 14.

Studies on the FH parameter between polybutadiene units of different vinyl content were performed by Sakurai et al. ${ }^{24}$ They assumed that there are three different interaction parameters to explain their experiment. One FH parameter describes the interaction between the monomer units of different microstructures, but with the same labeling (protonation or deuteration). The second $\mathrm{FH}$ parameter describes the interaction between monomer units of different microstructures and labelings. The third FH parameter describes the isotope effect between identical microstructures, which is an order of magnitude smaller. Despite this simplification the predicted FH parameter agrees well within a crosscheck. The enthal pic and entropic $\mathrm{FH}$ parameters of the h-PB $(1,2) / d-P B(1,4)$ blend measured in this work are larger by a factor of, respectively, 2.6 and 8.8 in comparison with the values of Sakurai. These discrepancies can be understood from a molecular weight dependence of the $\mathrm{FH}$ parameter coming from the chain ends and showing an additional $1 N$ term $\mathrm{m}^{25}$ and whose relative effect seems to be quite strong. ${ }^{26-28}$ Thus, the large difference of the $\mathrm{PB}(1,2) / \mathrm{PB}(1,4) \mathrm{FH}$ parameter as measured directly and as predicted by the intramolecuIar FH parameter of the PB/PS blend remains to be significant.

The $\mathrm{FH}$ parameters of polybutadiene blends with different vinyl contents $n$ were also discussed by Krishnamoorti et al. in ref 29. The theory predicts for the enthalpic portion of the $\mathrm{FH}$ parameter a dependence on the vinyl content difference $\Delta \varphi$, according to $\Gamma_{\mathrm{h}}(\mathrm{blend})$ $=(\Delta \varphi)^{2} \Gamma(1,2 ; 1,4)$. Experimentally, however, a more complicated dependence on the vinyl contents was found and, finally, the authors had to conclude that the model for statistical copolymers could at least not be applied to polybutadiene copolymer blends.

Lattice-cluster theory calculations on random copolymers yield a similar dependence of the entropic portion of the $\mathrm{FH}$ parameter due to the "monomer site occupancy index" and as formulated in eq $12 .{ }^{18}$ In the case of two polybutadiene components the proportionality $\Gamma_{\sigma}$ $\propto(\Delta \varphi)^{2}$ is predicted, which is the same as that in the theory of random copolymers. When d-PB/PS blends are regarded, a monotonic proportionality $\Gamma_{\sigma} \propto-(\varphi-1.5)^{2}$ is predicted. Neither prediction matches our experimental results. One reason might be a molar volume dependence of the $\mathrm{FH}$ parameter, which seems to be rather large for the $V_{w}=2200 \mathrm{~cm}^{3} / \mathrm{mol} P B(1,2) / d-P B-$ $(1,4)$ blend.26-28 One might further speculate that compressibility, chain semiflexibility, and specific interactions between the 1,2- and 1,4-butadiene monomers have to be included because they are neglected at the present stage of the lattice-cluster theory for pedestrians. ${ }^{18}$

Acknowledgment. M. Zähres from University of Duisburg and $\mathrm{M}$. Heiderich from our group are gratefully acknowledged for the NMR measurements and for the technical support during the SANS experiments, respectively.

\section{References and Notes}

(1) deGennes, P. G. Scaling Concepts in Polymer Physics; Cornell University Press: Ithaca, NY, 1979.

(2) Binder, K. Adv. Polym. Sci. 1994, 112, 181. 
(3) Higgins, J . S.; Benoit, H. Polymers and Neutron Scattering; Clarendon Press: Oxford, 1994

(4) Schwahn, D.; Mortensen, K. Thermal Composition Fluctuations in Polymer Blends studied with Small Angle Neutron Scattering. In Scattering in Polymeric and Colloidal Systems; Brown, W., Mortensen, K., Eds.; Gordon \& Breach: Langhorne, PA, 2000.

(5) Schwahn, D.; Meier, G.; Mortensen, K.; J anssen, S. J . Phys. II (France) 1994, 4, 837.

(6) Belyakov M. Y.; Kiselev S. B. Physica 1992, A190, 75

(7) Sengers, J. v. In Supercritical Fluids: Fundamentals for Application; Kiran, E., Levelt Sengers, J. M. H.; Kluwer Academic Publishers: Dordrecht, 1994.

(8) J anssen, S.; Schwahn, D.; Mortensen, K.; Springer, T. Macromolecules 1993, 26, 5587; Macromolecules 1995, 28, 2555.

(9) Hammouda, B.; Bauer, B. J . Macromolecules 1995, 29, 4505.

(10) Schwahn, D.; Schmackers, T.; Mortensen, K. Phys. Rev. E 1995, 52, R1288.

(11) Frielinghaus, H.; Abbas, B.; Schwahn, D.; Willner, L. Europhys. Lett. 1998, 44, 606.

(12) Ma, S. K. Statistical Mechanics; World Scientific: Singapore, 1982.

(13) Uzunov, D. I. Theory of Critical Phenomena; World Scientific: Singapore, 1993.

(14) Sariban, A.; Binder, K. J . Chem. Phys. 1987, 86, 5859.

(15) Paul, D. R.; Barlow, J. W. Polymer 1984, 25, 487.

(16) Wansoo, H.; Karasz, E. Macromolecules 1992, 25, 1057.
(17) Delfolie, C.; Dickinson, L. C.; Freed, K. F.; Dudowicz, J .; Macknight, W. J . Macromol ecules 1999, 32, 7781.

(18) Dudowicz, J .; Freed, K. F. Macromolecules 2000, 33, 3467.

(19) Koningsveld. R.; Chermin, H. A. G.; Gordon, M. Proc. R. Soc. London Ser. 1970, A319, 331.

(20) Neutronenstreuexperimenteam FRJ -2 in J ülich (the German and English text is available at Forschungzentrum J ülich, 1997).

(21) Morton, M.; Fetters, L. J . Rubber Chem. Technol. 1975, 48, 359.

(22) Marx-Figini, M.; Figini, R. V. Makromol. Chem. 1980, 181, 2401.

(23) Schwahn, D.; Mortensen, K.; J anssen, S. Phys. Rev. Lett. 1994, 73, 1452.

(24) Sakurai, S.; Hasegawa, H.; Hashimoto, T.; Hargis, I. G.; Aggarwal, S. L.; Han, C. C. Macromolecules 1990, 23, 451.

(25) Dudowicz, J .; Freed, K. Macromolecules 1993, 26, 213.

(26) Beaucage, G.; Sukumaran, S,; Claerson, S. J .; Kent, M. S.; Schaefer, D. Makromolecules 1986, 29, 8349.

(27) Frielinghaus, H.; Pedersen, W. P.; Larsen, P. S.; Almdal, K.; Mortensen, K. Makromolecules 2000 (submitted).

(28) Schwahn, D.; Willner, L. Corresponding experiments are in progress

(29) Krishnamoorti, R.; Graessley, W. W.; Fetters, L. J .; Garner, R. T.; Lohse, D. J . Macromolecules 1998, 31, 2312.

MA001015K 